\title{
EVM-Constrained and Mask-Compliant MIMO-OFDM Spectral Precoding
}

\author{
Shashi Kant, Student Member, IEEE, Mats Bengtsson, Senior Member, IEEE, Gabor Fodor, Senior Member, IEEE, \\ Bo Göransson, Member, IEEE, and Carlo Fischione, Senior Member, IEEE
}

\begin{abstract}
Spectral precoding is a promising technique to suppress out-of-band emissions and comply with leakage constraints over adjacent frequency channels and with mask requirements on the unwanted emissions. However, spectral precoding may distort the original data vector, which is formally expressed as the error vector magnitude (EVM) between the precoded and original data vectors. Notably, EVM has a deleterious impact on the performance of multiple-input multiple-output orthogonal frequency division multiplexing-based systems. In this paper we propose a novel spectral precoding approach which constrains the EVM while complying with the mask requirements. We first formulate and solve the EVM-unconstrained mask-compliant spectral precoding problem, which serves as a springboard to the design of two EVM-constrained spectral precoding schemes. The first scheme takes into account a wideband EVM-constraint which limits the average in-band distortion. The second scheme takes into account frequency-selective EVM-constraints, and consequently, limits the signal distortion at the subcarrier level. Numerical examples illustrate that both proposed schemes outperform previously developed schemes in terms of important performance indicators such as block error rate and systemwide throughput while complying with spectral mask and EVM constraints.
\end{abstract}

Index Terms-Sidelobe suppression, spectral precoding, MIMO, OFDM, EVM, out-of-band emissions, ACLR, Consensus ADMM, Douglas-Rachford Splitting.

\section{INTRODUCTION}

Modern wireless communication systems, including fifthgeneration (5G) New Radio (NR), adopt orthogonal frequency division multiplexing (OFDM) with cyclic prefix [2]. The reasons are that OFDM has several attractive characteristics such as robustness to the negative effects of time dispersive channels and multi-path fading, simplicity in terms of equalization and flexibility in terms of supporting both low and high symbol rates and thereby supporting a variety of quality of service requirements. Further, OFDM-based systems can facilitate dynamic spectrum sharing [3].

Unfortunately, OFDM suffers from high out-of-band emissions (OOBE) due to the discontinuities at the boundaries of the rectangular window and the high sidelobes associated with

S. Kant, B. Göransson, and G. Fodor are with Ericsson AB and KTH Royal Institute of Technology, Stockholm, Sweden (e-mail: \{shashi.v.kant, bo.goransson, gabor.fodor\} @ ericsson.com)

M. Bengtsson and C. Fischione are with KTH Royal Institute of Technology, Stockholm, Sweden (e-mail: \{mats.bengtsson@ee.kth.se, carlofi@kth.se\})

The work of S. Kant was supported in part by the Swedish Foundation for Strategic Research under grant ID17-0114.

Part of this paper on EVM-unconstrained OFDM spectral precoding was presented at IEEE SPAWC 2019 [1.

the sinc functions of the OFDM signal [4]. The OOBE must be adequately suppressed since high OOBE causes significant interference to the neighbouring adjacent channels. In practice, all OFDM systems are designed to comply not only with OOBE requirements, in terms of adjacent channel leakage ratio (ACLR) and spectral emission mask, but also in-band requirements, in terms of error vector magnitude (EVM) and other signal demodulation/detection requirements [5]. Simply, the EVM describes the distortion/noise incurred to the useful (transmit) symbols and ACLR represents the amount of undesired power that exists in the neighbouring carriers relative to the desired carrier power-the detailed description of these metrics are in the subsequent sections.

There are a plethora of techniques to suppress/reduce OOBE for the cyclic-prefix based OFDM-see, e.g., [6] and its references-which can be categorized into time and frequency domain methods. Amongst them, the methods are, namely, guard band inclusion, filtering [7], windowing [8], cancellation carriers [9]-[11], and spectral precoding [1], [12]-[18].

Spectral precoding is one of the promising bandwidth efficient techniques for OOBE reduction. It spectrally precodes the data symbols before OFDM modulation [13], [17], [18], which reduces the OOBE without using the extra spectral resources contrary to cancellation carriers and without increasing the delay/time dispersion or penalizing the cyclic prefix of the transmitted signal unlike filtering/windowing. In Section II we review related works in addition to our contribution.

In this paper, we develop spectral precoding schemes that comply with EVM-constraints and simultaneously meet OOBE requirements in terms of mask and ACLR, without affecting signal processing at the receiver. Our objective is to design low complexity spectral precoding algorithms that operate well in wide-band multiple-input multiple-output (MIMO)-OFDM systems. In the first part of the paper, we consider the problem of designing mask-compliant spectral precoding without EVM constraints. In the second part of the paper, we propose to incorporate wideband and frequencyselective EVM constraints in addition to mask compliance. Our goal is to improve key performance indicators, including out-of-band and in-band performance metrics, because highly spectral efficient MIMO-OFDM-based systems require low EVM. Accordingly, we introduce a wideband EVM constraint, which restricts the wideband average in-band distortion due to spectral precoding. Finally, the frequency-selective EVM constraint offers more flexibility-but at the expense of increased complexity - than using wideband constraints, because the frequency-selective constraints limit the in-band distortion

\footnotetext{
(a)
} 
power at the subcarrier (or group of subcarriers) level. As we will show and discuss, these design approaches represent at least three distinct ways of addressing the complexityperformance trade-off in the design of MIMO-OFDM spectral precoders.

The rest of the paper is structured as follows. The next section, which can be skipped by the reader familiar with the subject, discusses related work including impact of EVM and states our contribution. Section III discusses preliminaries and introduce the system model. Next, Section IV formulates the EVM-unconstrained problem and develops an alternating direction method of multipliers (ADMM)-based algorithm [19], [20] and a coordinate-descent-based algorithm to solve this unconstrained problem. Section $\mathrm{V}$ formulates the EVMconstrained problem and develops an ADMM-based solution and yet an alternative approach based on the Douglas-Rachford algorithm [19], [21]. Section VI presents simulation results that compare the performance of the proposed schemes with that of benchmarking schemes, and finally Section VII concludes the paper.

\section{RELATED WORKS AND CONTRIBUTIONS}

In this section, we first give an overview of the related frequency domain techniques, then the impact of EVM on the system performance, and finally highlight our original contribution.

\section{A. Cancellation Carrier Techniques}

The cancellation carrier techniques, such as active interference cancellation [9], cancellation carrier with power constraint [10], and extended active interference cancellation [11], utilize the non-data bearing subcarriers solely for OOBE reduction. These methods generally offer good sidelobe suppression with relatively low complexity, at the expense of transmit power wastage and spectral resources. Besides signalto-noise ratio (SNR) degradation due to transmit power sharing with the non-data bearing cancellation carriers, the extended active interference cancellation [11] induces intersymbol and intercarrier interferences to the desired/useful signal due to the placement of the non-orthogonal carriers. Additionally, there are several practical implementation challenges, e.g., many cancellation carriers are required to maintain appropriate power spectral density (PSD) of the composite signals to avoid the negative impact of intermodulation due to nonlinear components [22], e.g., power amplifier, and consequently failing to meet other OOBE requirements-in terms of mask and ACLR defined in NR-like standards [5].

\section{B. Spectral Precoding Techniques}

There are many variants of spectral precoding methods in the literature to suppress OOBE. We briefly review some of these techniques subsequently.

In the notching spectral precoder [13], the spectrally precoded signal essentially nulls/notches the OOBE at given discrete frequencies in the out-of-band spectrum of the OFDM signal. The precoder is obtained in the closed-form by solving equality constrained least-squares optimization problem.
Notching the spectrum at well-selected discrete frequencies often suppresses the whole signal spectrum and consequently leads to OOBE reduction [13]. However, such a notching approach has a deleterious impact on the in-band performance, in terms of increased EVM and block error rate, which thereby penalizes the system-wide throughput. Notably, the edge subcarriers have very high EVM-see Fig. 2)(c) in the numerical results Section VI-C-which decreases the throughput. Recognizing these problems, in [16] a weighted-notching spectral precoder method is proposed to reduce the EVM at the edge subcarriers by spreading the total distortion over all the allocated subcarriers. Another extension of the notching spectral precoder, proposed in [23], performs precoding jointly over several consecutive OFDM symbols which improves the in-band performance slightly over the single OFDM symbolbased precoding but penalizes the latency since a large number of OFDM symbols have to be buffered.

2) Constrained Spectral Precoding: On the contrary, maskcompliant spectral precoder (MSP) improves the in-band performance over notching spectral precoder while only meeting a target mask instead of creating nulls at the selected discrete frequencies at the expense of increased complexity [1], [24]. Because MSP is posed as an inequality constrained convex optimization formulation, it typically does not yield a closed-form solution. Furthermore, the authors of [15] suggest utilizing a generic optimization solver, which generally employs interior-point methods [25]. Therefore, the authors in [1], [24], propose computationally efficient schemes to obtain mask-compliant spectrally precoded data symbols for single-input single-output (SISO)-OFDM systems. The authors in [26] propose three EVM-constrained precoders for SISO systems, whereby two precoders are developed heuristically, and one of them is posed as a convex optimization problem. The optimization-based precoder is obtained by minimizing the $\ell_{2}$-norm of the OOBE at a chosen set of discrete frequency points subject to the EVM constraint-yielding no closedform solution. The former precoders are ad hoc, but can be seen as a scaled-form of the notching spectral precoder such that they meet the EVM constraint but penalize the OOBE in an unsystematic way. Furthermore, the authors show numerically that the ad hoc precoders render superior performance compared with the optimization-based precoder in suppressing OOBE under EVM constraint.

3) Spectral Precoding in MIMO Systems: In [27] several linear receivers are investigated when notching spectral precoders is employed in a MIMO-OFDM system. In [18, Paper F], the authors extend the SISO-OFDM spectral precoding to massive MIMO-OFDM for a joint spatial and notching spectral precoding, which exploits full downlink channel state information at the transmitter due to channel reciprocity in time division duplexing, to improve the in-band performance at the receiver. Recent works analyzed the impact of the EVM at the receiver or over-the-air specifically in massive MIMO context. These works reveal that in beamforming systems the EVM is also beamformed along the same direction as the data, see e.g., [28]-[31]. 


\section{Impact of EVM on the System Performance}

In practical transceivers, the hardware imperfections stem from both the digital and the analogue components. These may include clip noise, filter ripple or distortion, in-phase and quadrature (IQ) mismatch, non-linearity, local oscillator inducing phase noise, sampling clock offsets, timing and frequency error, see, e.g., [32], [33]. These imperfections have detrimental impact on the MIMO-OFDM performance, notably in high data rate achieving systems [34]-[39]. In practice, EVM metric evaluation provides insightful and useful information on the link quality, in terms of SNR, seen at the receiver due to the aggregated digital and analogue hardware imperfections [40]-[42]. The NR-like standards mandate (transmit) EVM requirements, see Table IV, on the composite inband distortion emanating from the various sources but measured at the (ideal) receiver by using pilots or reference signals as described in, e.g., [5, Appendix B].

To minimize the impact of EVM onto the system throughput performance, in general, there are at least two approaches: 1) constraining the EVM at the transmitter and 2) mitigating or cancelling the (transmit) EVM seen at the receiver. We pursue the former approach because NR-like standards [5], [43] stipulate the minimum EVM requirements according to the considered modulation alphabet, which must be satisfied by the base station or user equipment devices. Hence, in this paper, we constrain the EVM of spectral precoding in addition to mask compliance (which implicitly may ensure the fulfilment of minimum ACLR requirements).

\section{Contribution of the Paper}

In this paper, we design computationally efficient algorithms for the mask-compliant spectral precoding with (un)constrained EVM for the MIMO-OFDM-based systems that scale linearly with the number of supported transmit antennas and do not require additional signal processing at the receiver. More specifically,

- we propose and accomplish a wideband and frequencyselective EVM-constrained and mask-compliant spectral precoding formulation.

- we develop two highly efficient algorithms by decomposing the large-scale spectral precoding optimization problems for both unconstrained and constrained EVM into subproblems, where each subproblem yields closedform or efficient solution, which has a very low computational complexity compared to the general purpose solver. We propose solutions for (un)constrained EVM: 1) ADMM-based algorithms, referred to as ADMM/EVMconstrained ADMM (EADMM) and 2) specialized algorithms, dubbed as semi-analytical spectral precoding (SSP)/EVM-constrained SSP (ESSP). Note that part of [1] is used for the EVM-unconstrained part of the present paper.

- we finally present exhaustive simulations using a 5G NR (Release-15 compliant) inhouse link-level simulator [1].

\section{E. Notation}

Let the set of complex and real numbers be denoted by $\mathbb{C}$ and $\mathbb{R}$, respectively. $\Re\{x\}$ denotes the real part of a complex number $x$. The $i$-th element of a vector $\boldsymbol{a} \in \mathbb{C}^{m \times 1}$ is denoted by $a[i] \in \mathbb{C}$, and element in the $i$-th row and $j$-th column of the matrix $\boldsymbol{A} \in \mathbb{C}^{m \times n}$ is denoted by $\boldsymbol{A}[i, j] \in \mathbb{C}$. The $i$-th row and $j$-th column vector of a matrix $\boldsymbol{A} \in \mathbb{C}^{m \times n}$ are represented as $\boldsymbol{A}[i,:] \in \mathbb{C}^{1 \times n}$ and $\boldsymbol{A}[:, j] \in \mathbb{C}^{m \times 1}$, respectively. An $i$-th higher order vector and matrix are denoted as $\boldsymbol{x}[i] \in \mathbb{C}^{m \times 1}$ or $\boldsymbol{x}_{i} \in \mathbb{C}^{m \times 1}$ and $\boldsymbol{X}[i] \in \mathbb{C}^{m \times n}$. We form a matrix by stacking the set of higher order vectors $\left\{\boldsymbol{a}[n] \in \mathbb{C}^{M \times 1}\right\}_{n=1}^{N}$ and $\left\{\boldsymbol{b}[m] \in \mathbb{C}^{1 \times N}\right\}_{m=1}^{M}$ columnwise and row-wise as $\boldsymbol{A}=[\boldsymbol{a}[1], \ldots, \boldsymbol{a}[N]] \in \mathbb{C}^{M \times N}$ and $\boldsymbol{B}=[\boldsymbol{b}[1] ; \ldots ; \boldsymbol{b}[M]] \in \mathbb{C}^{M \times N}$, respectively. The dimensions of the vectors/matrices are equally applicable for both $\mathbb{C}$ and $\mathbb{R}$. The transpose and conjugate transpose of a vector or matrix are denoted by $(\cdot)^{\mathrm{T}}$ and $(\cdot)^{\mathrm{H}}$, respectively. The complex conjugate is represented by $(\cdot)^{*}$. The $K \times K$ identity matrix is written as $\boldsymbol{I}_{K}$. The expectation operator is denoted by $\mathbb{E}\{\cdot\}$. An $i$-th iterative update is denoted by $(\cdot)^{(i)}$.

\section{PRELIMINARIES}

In this section, we introduce the downlink MIMO-OFDM system model followed by performance metrics useful for the spectral precoding design.

\section{A. System Model and Out-of-Band Emissions}

We consider the OFDM-based single-user MIMO downlink, where the base station is equipped with $N_{\mathrm{T}}$ transmit (Tx) antennas, and the user equipment (UE) is equipped with $N_{\mathrm{R}}$ receive $(\mathrm{Rx})$ antennas as depicted in Fig. 1 Additionally, we reckon spatial multiplexing transmission scheme with $N_{\mathrm{L}} \leq$ $\min \left\{N_{\mathrm{T}}, N_{\mathrm{R}}\right\}$ spatial layers.

A spatially precoded symbol vector $\boldsymbol{x}[k]$ at $k$-th subcarrier for a given OFDM symbol can be formed by

$$
\boldsymbol{x}[k]=\boldsymbol{W}[k] \boldsymbol{s}[k] \in \mathbb{C}^{N_{\mathrm{T}} \times 1},
$$

where, the vector $s[k] \in \mathcal{S}^{N_{\mathrm{L}} \times 1}$ belongs to a complexvalued finite-alphabet set $\mathcal{S}, e . g$., corresponding to a $2^{Q_{-}}$ quadrature amplitude modulation (QAM) constellation with $Q \in\{2,4,6,8\}$. The given MIMO precoder $\boldsymbol{W}[k] \in \mathbb{C}^{N_{\mathrm{T}} \times N_{\mathrm{L}}}$ is chosen, e.g., from a codebook in 3GPP NR [44].

Towards this end, we introduce a frequency-domain (spatially-precoded) data matrix $\boldsymbol{X}$ by stacking each (column) vector $\boldsymbol{x}[k]$ (cf. Eq. (1) column-wise for all the subcarriers within a given $N$-point inverse discrete Fourier transform such that

$$
\boldsymbol{X}:=[\boldsymbol{x}[1], \ldots, \boldsymbol{x}[N]]:=\left[\boldsymbol{d}_{1}^{\mathrm{T}} ; \ldots ; \boldsymbol{d}_{N_{\mathrm{T}}}^{\mathrm{T}}\right] \in \mathbb{C}^{N_{\mathrm{T}} \times N},
$$

where we define a spatially precoded vector $\boldsymbol{d}_{j} \in \mathbb{C}^{N \times 1}$ for $j$-th transmit antenna as

$$
\boldsymbol{d}_{j}:=(\boldsymbol{X}[j,:])^{\mathrm{T}} \in \mathbb{C}^{N \times 1} .
$$

Similarly, we define a spectrally precoded frequency-domain data matrix $\overline{\boldsymbol{X}}$ as

$$
\overline{\boldsymbol{X}}:=[\overline{\boldsymbol{x}}[1], \ldots, \overline{\boldsymbol{x}}[N]]:=\left[\overline{\boldsymbol{d}}_{1}^{\mathrm{T}} ; \ldots ; \overline{\boldsymbol{d}}_{N_{\mathrm{T}}}^{\mathrm{T}}\right] \in \mathbb{C}^{N_{\mathrm{T}} \times N},
$$

where the spectrally (and spatially) precoded data symbol at $k$-th subcarrier is represented as $\overline{\boldsymbol{x}}[k]$. Further, we define a column vector $\overline{\boldsymbol{d}}_{j} \in \mathbb{C}^{N \times 1}$ of a spectrally precoded vector corresponding to $j$-th transmit antenna branch as

$$
\overline{\boldsymbol{d}}_{j}:=(\overline{\boldsymbol{X}}[j,:])^{\mathrm{T}} \in \mathbb{C}^{N \times 1} \text {. }
$$




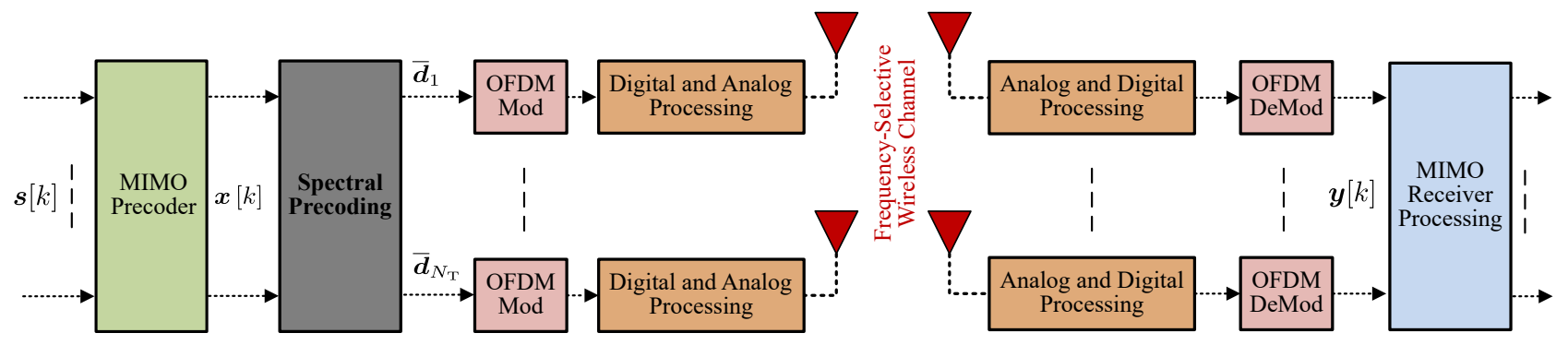

Fig. 1: Simplified block diagram of a single-user MIMO-OFDM transceiver with spectral precoding.

\section{B. Performance Metrics}

We utilize two figure-of-merits, i.e., OOBE and inband distortions, for the spectral precoding design and its performance. The inband distortions are evaluated in terms of EVM and also block error rate (BLER) (or equivalently throughput). More specifically, we have employed EVM metric for the spectral precoding design. Nonetheless, there exists a nonlinear mapping between EVM and BLER or equivalently received SNR, see, e.g., [41], [45]. Therefore, EVM metric is sufficient for the spectral precoding design and performance evaluation. The OOBE requirements are typically characterized by the spectral mask and ACLR, among others [5], [43]. Based on our practical experience, if the base station fulfils the spectral mask requirement with a suitable implementation margin then the ACLR requirement is also achieved; and the converse is also true. Therefore, we have solely considered an appropriately discretized mask as a spectral precoding design parameter. However, for the numerical performance evaluation, we also evaluate ACLR for completeness.

1) Out-of-Band Emissions: The OOBE is typically quantified in terms of the operating band unwanted emissions and (conducted) ACLR, whose definitions are given below.

Definition 1 (Operating band unwanted emissions [5, Section 6.6.4], referred to as mask). Unwanted emissions that are immediately outside the base station channel bandwidth resulting from the modulation process and non-linearity in the transmitter but excluding spurious emissions.

The unwanted OOBE due to the OFDM frequency-domain signal $\boldsymbol{d}_{j}$ at the $M$ considered discrete frequency points $\boldsymbol{\nu}=$ $\left[\nu_{1}, \ldots, \nu_{M}\right]$ can be described by $\boldsymbol{p}(\boldsymbol{\nu})=\boldsymbol{A} \boldsymbol{d}_{j}$.

We now define $\boldsymbol{A}[m,:]:=\boldsymbol{a}\left(\nu_{m}\right)^{\mathrm{T}} \in \mathbb{C}^{1 \times N}$, where $\boldsymbol{A}[m, k]:=a\left(\nu_{m}, k\right)$ can be derived in discrete form as [13]:

$$
\begin{aligned}
a\left(\nu_{m}, k\right)= & \left(\frac{1}{\sqrt{N}}\right) \exp \left(j \pi \frac{\left(\nu_{m}-k\right)}{N}\left(N_{\mathrm{CP}}-N+1\right)\right) . \\
& \cdot \frac{\sin \left(\pi \frac{\left(\nu_{m}-k\right)}{N}\left(N+N_{\mathrm{CP}}\right)\right)}{\sin \left(\pi \frac{\left(\nu_{m}-k\right)}{N}\right)}
\end{aligned}
$$

where $N_{\mathrm{CP}}$ corresponds to cyclic prefix length in samples.

Definition 2 (Adjacent channel leakage ratio (ACLR) [5, Section 6.6.3]). ACLR is the ratio of the filtered mean power centred on the assigned channel frequency to the filtered mean power centred on an adjacent channel frequency. The (worstcase) ACLR can mathematically be expressed as

$$
\operatorname{ACLR}:=\frac{\int_{-\mathrm{BW} / 2}^{\mathrm{BW} / 2} S_{\text {desiredchannel }}(f) d f}{\max \left\{\int_{\frac{-3 \mathrm{BW}}{2}}^{\frac{-\mathrm{BW}}{2}} S_{\text {leftchannel }}(f) d f, \int_{\frac{\mathrm{BW}}{2}}^{\frac{3 \mathrm{BW}}{2}} S_{\text {rightchannel }}(f) d f\right\}},
$$

where $S_{\text {desiredchannel }}$ is the PSD in the desired carrier having BW bandwidth including the guard band; and similarly, $S_{\text {leftchannel }}$ and $S_{\text {rightchannel correspond to the PSD on the }}$ left and the right side of the desired carrier having same bandwidth $\mathrm{BW}$ as the desired carrier, respectively.

We would like to accentuate that we do not directly use ACLR for the spectral precoding design, but rather we use the OOBE power at the considered discrete frequency points.

2) In-Band Distortion: The considered in-band distortion for the spectral precoding design is EVM, which can be quantified as a loss in the demodulated signal quality. It can be described mathematically per $j$-th transmit antenna as 1

$$
\mathrm{EVM}_{j}:=\boldsymbol{\epsilon}_{j}=\frac{\mathbb{E}\left\{\left\|\boldsymbol{d}_{j}-\overline{\boldsymbol{d}}_{j}\right\|_{2}^{2}\right\}}{\mathbb{E}\left\{\left\|\boldsymbol{d}_{j}\right\|_{2}^{2}\right\}} .
$$

For the spectral precoding design, we assume that the average transmit signal power per transmit antenna branch $\mathbb{E}\left\{\left\|\boldsymbol{d}_{j}\right\|_{2}^{2}\right\}$ is fixed.

\section{EVM-UNCONSTRAINED LARGE-SCALE MSP (LS-MSP) IN MIMO-OFDM}

In this section, we first consider a previously proposed mask-compliant spectral precoding without EVM constraint that utilizes a generic convex optimization solver. It is known that it suffers from high computational complexity, notably in large-scale systems. Afterwards, to mitigate the complexity of computing the LS-MSP, we propose a divide-andconquer approach that breaks the original problem into smaller rank 1 quadratic-constraint problems, where each small problem yields a closed-form solution. Lastly, based on these solutions, we develop two specialized first-order low-complexity algorithms. In particular, the first one is based on the consensus ADMM while the second one is derived by employing the coordinate descent scheme of a dual variable and capitalizing on closed-form of the rank 1 constraint.

\footnotetext{
${ }^{1} \mathrm{EVM}$ measurements in NR standard are stipulated with a zero-forcing receiver, see, e.g., [5. Annex B], which can be seen as an equalized EVM measure. On contrast, the EVM for the spectral precoding design can be defined as an unequalized EVM since the unequalized EVM value would be conservative, i.e., it can not be less than the equalized EVM, see, e.g., [46].
} 


\section{A. EVM-Unconstrained Proposed Problem Formulations}

The work in [13] was extended in [15] for single-antenna OFDM, referred to as MSP, such that only the mask constraint needs to be fulfilled, i.e.,

$$
\underset{\overline{\boldsymbol{d}}_{j} \in \mathbb{C}^{N \times 1}}{\operatorname{minimize}}\left\|\boldsymbol{d}_{j}-\overline{\boldsymbol{d}}_{j}\right\|_{2}^{2} \quad \text { subject to }\left|\boldsymbol{A} \overline{\boldsymbol{d}}_{j}\right|^{2} \preceq \boldsymbol{\gamma},
$$

where the inequality constraint is element-wise and the target mask $\gamma \in \mathbb{R}^{M \times 1}$ is given. The solution could not be expressed in (semi) closed-form and thereby the authors proposed to solve this MSP problem via a generic quadratic programming solver [15], e.g., CVX [47].

Based on our key observation, we rewrite the problem (8) as described below, without any loss of convexity. More specifically, the constraint in 8 can be decomposed into $M$ rank 1 constraints such that it becomes

$$
\begin{array}{ll}
\underset{\overline{\boldsymbol{d}}_{j}}{\operatorname{minimize}} & \left\|\boldsymbol{d}_{j}-\overline{\boldsymbol{d}}_{j}\right\|_{2}^{2} \\
\text { subject to } & \overline{\boldsymbol{d}}_{j}^{\mathrm{H}} \overline{\boldsymbol{A}}_{m} \overline{\boldsymbol{d}}_{j} \leq \gamma_{m}, \forall m=1 \ldots, M,
\end{array}
$$

where

$$
\overline{\boldsymbol{A}}_{m}=\boldsymbol{a}\left(\nu_{m}\right)^{*} \boldsymbol{a}\left(\nu_{m}\right)^{\mathrm{T}} \in \mathbb{C}^{N \times N}
$$

and $\operatorname{rank}\left\{\overline{\boldsymbol{A}}_{m}\right\}=1$. Problem (9) is referred to as LS-MSP that facilitates large-scale optimization.

\section{B. Efficient Algorithms for EVM-Unconstrained LS-MSP}

The proximal operator, prox, is used for the algorithm design, whose definition is given below.

Definition 3 (proximal mapping [48], [49]). Given a proper closed convex function $f: \operatorname{dom}_{\mathrm{f}} \mapsto(-\infty,+\infty]$, then the proximal mapping of $f$ is the operator given by

$$
\operatorname{prox}_{\lambda f}(\boldsymbol{x})=\arg \min _{\boldsymbol{z} \in \operatorname{dom}_{\mathrm{f}}}\left\{f(\boldsymbol{z})+\frac{1}{2 \lambda}\|\boldsymbol{x}-\boldsymbol{z}\|_{2}^{2}\right\}
$$

for any $\boldsymbol{x} \in \operatorname{dom}_{\mathrm{f}}$, where $\operatorname{dom}_{\mathrm{f}}$ corresponds to the domain of function $f$ and $\lambda>0$.

Definition 4 (proximal mapping of the indicator function [48], [49]). Let $f: \operatorname{dom}_{\mathrm{f}} \mapsto(-\infty,+\infty]$ be an indicator function, $f(\boldsymbol{x}):=X_{\mathcal{C}}(\boldsymbol{x})$ where $C$ is a nonempty set $X_{\mathcal{C}}(\boldsymbol{x})=0$ if $\boldsymbol{x} \in \mathcal{C}$ otherwise $X_{C}(\boldsymbol{x})=+\infty$, then the proximal mapping of a given set $\mathcal{C}$ is an orthogonal projection operator $\operatorname{proj}_{\mathcal{C}}$ onto the same set, i.e.,

$$
\begin{aligned}
\operatorname{prox}_{\lambda x_{\mathcal{C}}}(\boldsymbol{x}) & =\arg \min _{\boldsymbol{z} \in \operatorname{dom}_{\mathrm{f}}}\left\{x_{\mathcal{C}}(\boldsymbol{x})+\frac{1}{2 \lambda}\|\boldsymbol{x}-\boldsymbol{z}\|_{2}^{2}\right\} \\
& =\arg \min _{\boldsymbol{z} \in \mathcal{C}}\left\{\frac{1}{2}\|\boldsymbol{x}-\boldsymbol{z}\|_{2}^{2}\right\}=\operatorname{proj}_{\mathcal{C}}(\boldsymbol{x}) .
\end{aligned}
$$

If $M=1$, the orthogonal projection onto the rank 1 quadratic constraint is obtained in closed-form as described in the following theorem:

Theorem 1 (projection onto the rank 1 quadratic constraint). Let $C \subseteq \mathbb{C}^{N \times 1}$ and $C \neq \emptyset$ given by $C=$ $\left\{\boldsymbol{x} \in \mathbb{C}^{N \times 1}: \boldsymbol{x}^{\mathrm{H}} \widetilde{\widetilde{A}} \boldsymbol{x}-b \leq 0\right\}$, where $\widetilde{\boldsymbol{A}}=\boldsymbol{u} \boldsymbol{u}^{\mathrm{H}} \in \mathbb{C}^{N \times N}$ is rank 1 matrix and $b \in \mathbb{R}_{\geq 0}$, then the proximal operator

$$
\operatorname{prox}_{X_{\mathcal{C}}}(x)=\operatorname{proj}_{\mathcal{C}}(x)
$$

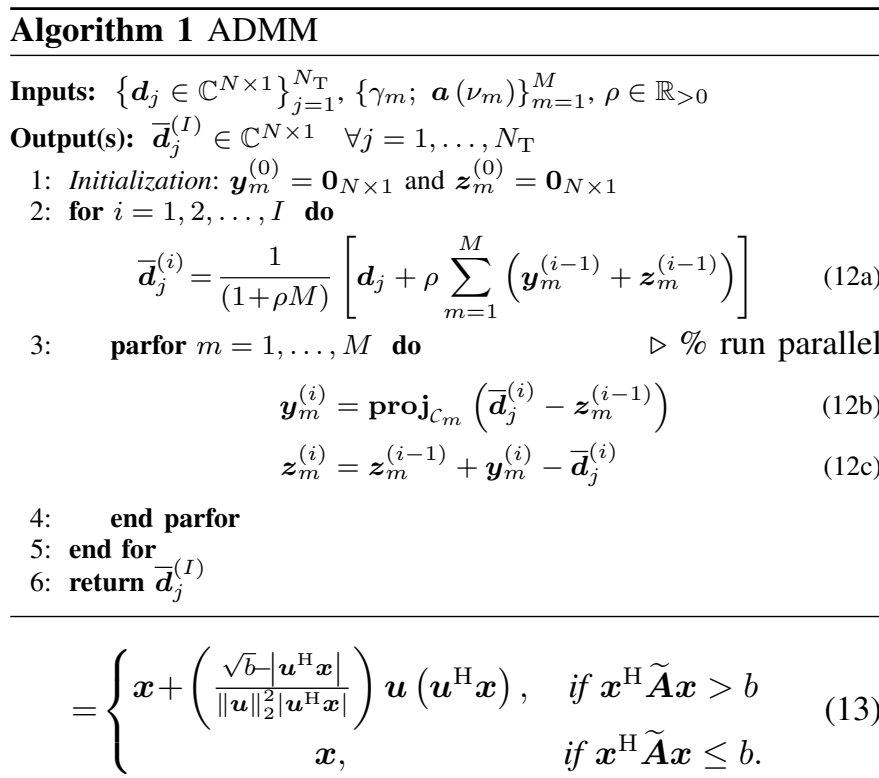

Proof. See Appendix B

If $M>1$, then no closed-form is known yet. Hence, in the sequel, we propose low-complexity algorithms that essentially break down the LS-MSP problem into smaller subproblems, where each subproblem admits closed-form solution capitalizing on Theorem 1

1) EVM-Unconstrained ADMM LS-MSP (referred to as $A D M M$ ): In our first proposal, we utilize ADMM with consensus optimization to solve the LS-MSP problem by rewriting Problem 9

$$
\begin{array}{ll}
\underset{\overline{\boldsymbol{d}}_{j}, \boldsymbol{y}_{m} \in \mathbb{C}^{N \times 1}}{\operatorname{minimize}} & f\left(\overline{\boldsymbol{d}}_{j}\right)+\sum_{m=1}^{M} x_{\mathcal{C}_{m}}\left(\boldsymbol{y}_{m}\right) \\
\text { subject to } & \boldsymbol{y}_{m}=\overline{\boldsymbol{d}}_{j} \quad \forall m=1, \ldots, M,
\end{array}
$$

where the (non-equalized) squared EVM $f\left(\overline{\boldsymbol{d}}_{j}\right)$ := $\left\|\boldsymbol{d}_{j}-\overline{\boldsymbol{d}}_{j}\right\|_{2}^{2}$ is a convex and differentiable function. The non-differentiable indicator function $x_{\mathcal{C}_{m}}\left(\boldsymbol{y}_{m}\right)$ with the rank 1 constraint set is given by $\mathcal{C}_{m}=\left\{\boldsymbol{y}_{m}: \boldsymbol{y}_{m}^{\mathrm{H}} \overline{\boldsymbol{A}}_{m} \boldsymbol{y}_{m}-\gamma_{m} \leq 0\right\}$.

Algorithm 1 summarizes the proposed recipe for the ADMM-based spectral precoding, cf. [1] for details, where $I$ denotes the total number of iterations. The convergence analysis of the ADMM algorithm is given in Appendix A

2) EVM-Unconstrained SSP LS-MSP (referred to as SSP): In our second proposal, we derive an optimal semi-analytical algorithm, dubbed as SSP, based on the Karush-Kuhn-Tucker (KKT) conditions [25] for the constrained optimization 96.

We form the Lagrangian of (9) by introducing the Lagrange multipliers $\left\{\mu_{m}\right\}$ as follows:

$$
L\left(\overline{\boldsymbol{d}}_{j},\left\{\mu_{m}\right\}\right)=\left\|\boldsymbol{d}_{j}-\overline{\boldsymbol{d}}_{j}\right\|_{2}^{2}+\sum_{m=1}^{M} \mu_{m}\left(\overline{\boldsymbol{d}}_{j}^{\mathrm{H}} \overline{\boldsymbol{A}}_{m} \overline{\boldsymbol{d}}_{j}-\gamma_{m}\right) .
$$

Utilizing the KKT conditions, the stationarity condition yields (15c) and the Lagrange multipliers $\left\{\mu_{m}\right\}$ are obtained iteratively in a coordinate descent fashion [50] as outlined in Algorithm 2 - see Appendix C for a detailed derivation. 
TABLE I: Comparison of online complexity of various EVM-unconstrained and mask-compliant algorithms

\begin{tabular}{|l||c|c|}
\hline Method & Complexity: Real Multiplications & Complexity: Real Additions \\
\hline MSP $[15]$ & $\mathcal{O}\left(N^{4.5} N_{\mathrm{T}}\right)[24]$ & - \\
\hline ADMM & $I(9 M N+M+N+1) N_{\mathrm{T}}$ & $I(14 M N+2 N+M+1) N_{\mathrm{T}}$ \\
\hline SSP & $\left(\left[5 M N+I\left(4 M^{2} N^{2}+12 M N^{2}+5 M^{2} N+3 M N+M\right)\right]\right) N_{\mathrm{T}}$ & $\left(\left[2 M N+I\left(2 M^{2} N^{2}+6 M N^{2}+6 M^{2} N-2 M N+M^{2}\right)\right]\right) N_{\mathrm{T}}$ \\
\hline
\end{tabular}

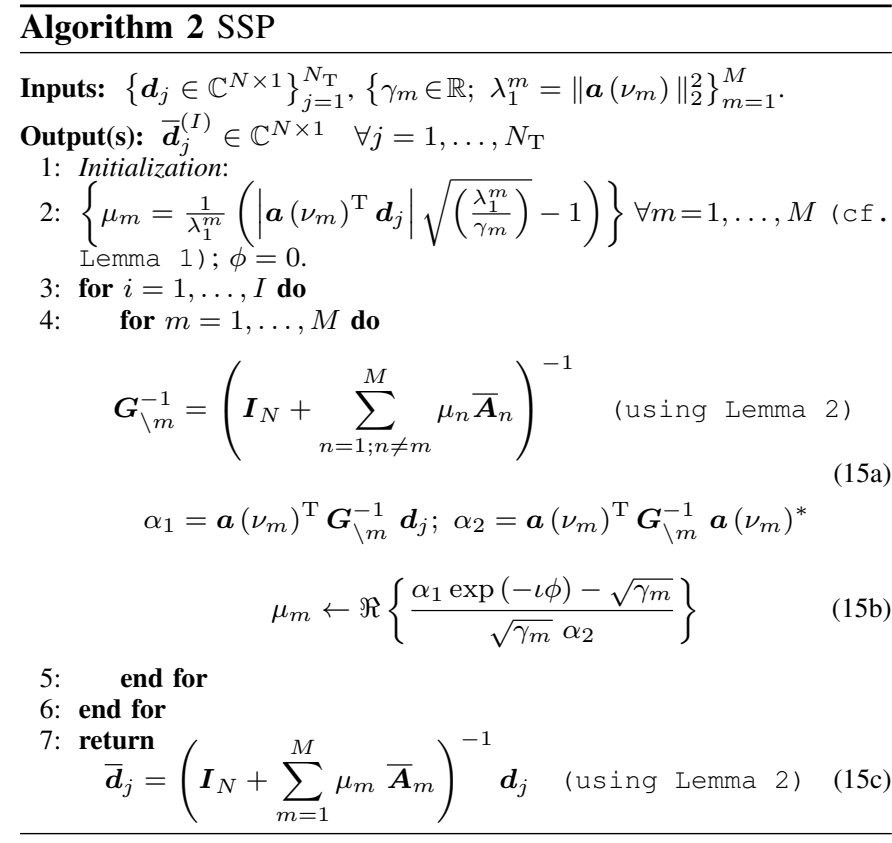

\section{Complexity Analysis}

We analyze the run-time complexity, in terms of required real-valued multiplications and real-valued additions but ignore the offline complexity. Notice, we convert all the complex multiplications and additions into equivalent real-valued multiplications and additions, i.e., 1 complex multiplication is equivalent to 4 real multiplications and 2 real additions, and 1 complex addition corresponds to 2 real additions. Furthermore, we assume that all the subcarriers are allocated, which will give the worst-case complexity analysis. Table I summarizes the complexity of the mask-compliant precoding schemes.

ADMM: The initialization step requires no multiplications/additions. In step $12 \mathrm{a}$, there are $(M+1) N$ complex additions, $N+1$ real multiplications, and 1 real addition per iteration and transmit antenna. The dominating online algebraic complexity is in the computation of the prox operator in step (12b), which is in the order of $2 M N$ complex multiplications, $2 M N$ complex additions, $M(N+1)$ real multiplications, and $M$ real additions per iteration and transmit antenna. However, due to distributed nature of consensus ADMM, the $M$ subiterations can run in parallel per iteration cycle at the expense of increased memory requirements. In step $12 \mathrm{c}$, we need $2 M N$ complex additions. Thus, ignoring parallelization, the total run-time complexities for $I$ iterations and $N_{\mathrm{T}}$ transmit antennas are $I(9 M N+M+N+1) N_{\mathrm{T}}$ and $I(14 M N+2 N+M+1) N_{\mathrm{T}}$ in terms of real multiplications and real additions, respectively.

SSP: The initialization step needs $N$ complex and real multiplications, respectively for each $m$-th frequency point and antenna, where $\left\{\lambda_{1}^{m}\right\}$ can be computed offline. The main computational complexity of step 15a is due to the matrix inversion, but no online inversion is necessary due to the sum of rank 1 matrices-see Lemma 2, which are $(M-1) N^{2}+N M$ complex multiplications, $2(M-1) N$ complex additions, $(M-1) N$ real multiplications, and $(M-1)$ real additions for each frequency point, iteration, and transmit antenna. The complexity of the computation of both $\alpha_{1}$ and $\alpha_{2}$ are $\left(2 N^{2}\right)$ for each frequency point, iteration, and transmit antenna. The step (15b) need $N$ complex multiplications, 1 real multiplication, and 1 real addition. Hence, the total online real multiplications and real additions for $I$ iterations and $N_{\mathrm{T}}$ transmit antennas are $\left(\left(\left[5 M N+I\left(4 M^{2} N^{2}+12 M N^{2}+5 M^{2} N+3 M N+M\right)\right]\right) N_{\mathrm{T}}\right)$ and $\left(\left(\left[2 M N+I\left(2 M^{2} N^{2}+6 M N^{2}+6 M^{2} N-2 M N+M^{2}\right)\right]\right) N_{\mathrm{T}}\right)$, respectively.

\section{EVM-CONSTRAINED LS-MSP IN MIMO-OFDM}

In this section, we firstly extend the LS-MSP Problem (9) such that the spectrally precoded symbol yearns to keep the EVM below the desired level by sacrificing OOBE performance in terms of ACLR, referred to as EVM-constrained MSP (EMSP). Unfortunately, adding EVM constraint in addition to the mask constraint poses challenges to develop a computationally efficient algorithm. Thanks to ADMM, which offers a divide-and-conquer approach, incorporating EVM constraint becomes easy and the algorithm is referred to as EADMM. However, SSP becomes prohibitively complex to support EVM constraint. Therefore, we have proposed an alternative operator splitting framework based on the DouglasRachford algorithm [19], [21], referred to as EVM-constrained SSP (ESSP), that employs the iterative SSP algorithm internally for the mask constraint and the outer loop of DouglasRachford supports the EVM constraint.

\section{A. EVM-Constrained Proposed Problem Formulations}

We pose wideband and frequency-selective EVM constrained mask-compliant spectral precoding optimization problems and then develop low-complexity algorithms. We firstly perform the epigraph transformation [51] of Problem (9), without losing convexity, such that the proposed wideband EVM-constrained optimization problem reads as:

$$
\begin{array}{ll}
\underset{\overline{\boldsymbol{X}} \in \mathbb{C}^{N_{\mathrm{T}} \times N}, \Delta t \in \mathbb{R}}{\operatorname{minimize}} & \Delta t \\
\text { subject to } & \|\overline{\boldsymbol{X}}-\boldsymbol{X}\|_{F} \leq \epsilon_{\mathrm{avg}} \\
& \left|\boldsymbol{A} \overline{\boldsymbol{X}}^{\mathrm{T}}\right|^{2} \preceq \boldsymbol{\Gamma} \odot \Delta t \mathbf{1}_{M \times N_{\mathrm{T}}},
\end{array}
$$

where $\odot$ represents element-wise multiplication, and $\epsilon_{\mathrm{avg}}$ denotes the desired wideband averaged EVM over all the allocated subcarriers (frequency-domain) and also over all the 
transmit antennas ${ }^{2}$, The target mask is $\Gamma \in \mathbb{R}^{M \times N_{\mathrm{T}}}$, e.g., $\boldsymbol{\Gamma}[:, j]=\left[\gamma_{1} ; \ldots ; \gamma_{m}\right]$ and $\mathbf{1}_{M \times N_{\mathrm{T}}}$ is an all-ones $M \times N_{\mathrm{T}}$ matrix. Note that the mask constraints can be the same for all the transmit antenna branches. Furthermore, the constraint (16c) can be decomposed for each $j$-th transmit antenna and each $m$-th discrete frequency point such that the constraint can be read as $\overline{\boldsymbol{d}}_{j}^{\mathrm{H}} \overline{\boldsymbol{A}}_{m} \overline{\boldsymbol{d}}_{j} \leq \Delta t \gamma_{m}$.

To support more flexibility in terms of defining different EVM constraints to different subcarriers, we extend the wideband EVM-constrained Problem (16) that offers different EVM constraint for each subcarrier. A frequency-selective EVM-constrained optimization problem is

$$
\begin{array}{ll}
\underset{\overline{\boldsymbol{X}} \in \mathbb{C}^{N_{\mathrm{T}} \times N}, \Delta t \in \mathbb{R}}{\operatorname{minimize}} & \Delta t \\
\text { subject to } & \|\overline{\boldsymbol{X}}[:, k]-\boldsymbol{X}[:, k]\|_{2} \leq \boldsymbol{\epsilon}[k] \forall k \in \mathcal{T} \\
& \left|\boldsymbol{A} \overline{\boldsymbol{X}}^{\mathrm{T}}\right|^{2} \preceq \boldsymbol{\Gamma} \odot \Delta t \mathbf{1}_{M \times N_{\mathrm{T}},}
\end{array}
$$

where $\epsilon[k]$ is the desired EVM at $k$-th subcarrier and the set $\mathcal{T}$ denotes all activated subcarriers.

The main benefit of Problem (17) is that the EVM constraint per subcarrier or the group of subcarriers can be defined by the upper layers depending on the channel link quality and/or the allocation of the data/pilots appropriately. In other words, such frequency-selective EVM constraint may have a high threshold (or high allowable EVM) for the lower supported modulation alphabet, e.g., for QPSK modulation - cf. Table IV] In contrast, the EVM constraint may have a low threshold for the higher modulation alphabet, e.g., for 64QAM.

An optimal solution to both problems (16) and (17) can be obtained via a general purpose optimization solver, e.g., CVX [47]. However, as motivated in the previous section, such general purpose algorithms employ interior-point-based methods whose complexity is prohibitively high. Hence, in order to develop efficient algorithms for the EVM-constrained and mask-compliant spectral precoding problems, we now instead transform the problems (16) and (17) into the feasibility problems by omitting the $\Delta t$ variable, i.e., problems (18) and (19), respectively. If $\Delta t \leq 1$ in problems (16) and (17), then the respective problems (18) and (19) are feasible. In other words, we make an assumption that the problem is feasible or has at least one solution, which implies $\Delta t \leq 1$, then the mask constraint (16c) can be expressed as following:

$$
\left|\boldsymbol{A} \overline{\boldsymbol{X}}^{\mathrm{T}}\right|^{2} \preceq \boldsymbol{\Gamma} \odot \Delta t \mathbf{1}_{M \times N_{\mathrm{T}}} \stackrel{\Delta t \leq 1}{\preceq} \boldsymbol{\Gamma} .
$$

Consequently, we omit $\Delta t$ from the the mask constraint in 166/177 for the feasibility problem. A wideband EVM constraint Problem (16) can be posed as the following feasibility problem:

$$
\begin{array}{ll}
\text { find } & \overline{\boldsymbol{X}} \in \mathbb{C}^{N_{\mathrm{T}} \times N} \\
\text { subject to } & \|\overline{\boldsymbol{X}}-\boldsymbol{X}\|_{F} \leq \epsilon_{\text {avg }} \\
& \left|\boldsymbol{A} \overline{\boldsymbol{X}}^{\mathrm{T}}\right|^{2} \preceq \boldsymbol{\Gamma} .
\end{array}
$$

Similarly, Problem [17, i.e., a frequency-selective EVM constraint with mask-compliant problem, can be posed as the

\footnotetext{
${ }^{2}$ It is straightforward to modify the problem to support wideband EVMconstraint per transmit antenna branch.
}

following feasibility problem:

$$
\begin{array}{ll}
\text { find } & \overline{\boldsymbol{X}} \in \mathbb{C}^{N_{\mathrm{T}} \times N} \\
\text { subject to } & \|\overline{\boldsymbol{X}}[:, k]-\boldsymbol{X}[:, k]\|_{2} \leq \boldsymbol{\epsilon}[k] \quad \forall k \in \mathcal{T} \\
& \left|\boldsymbol{A} \overline{\boldsymbol{X}}^{\mathrm{T}}\right|^{2} \preceq \boldsymbol{\Gamma} .
\end{array}
$$

Now, the respective mask and wideband and frequencyselective EVM constraint sets are defined as following. The $m$-th mask constraint set corresponds to the rank 1 quadratic inequality, i.e.,

$$
\mathcal{C}_{m}:=\left\{\overline{\boldsymbol{X}}: \overline{\boldsymbol{d}}_{j}^{\mathrm{H}} \overline{\boldsymbol{A}}_{m} \overline{\boldsymbol{d}}_{j}-\gamma_{m} \leq 0 ; \forall j=1, \ldots, N_{\mathrm{T}}\right\},
$$

and the wideband EVM constraint set can be described by

$$
\mathcal{E}_{\mathrm{wb}}:=\left\{\overline{\boldsymbol{X}}:\|\overline{\boldsymbol{X}}-\boldsymbol{X}\|_{F}-\epsilon_{\mathrm{avg}} \leq 0\right\},
$$

whereas the frequency-selective EVM set can be expressed as

$$
\mathcal{E}_{\mathrm{fs}}:=\left\{\overline{\boldsymbol{X}}:\|\overline{\boldsymbol{X}}[:, k]-\boldsymbol{X}[:, k]\|_{2}-\boldsymbol{\epsilon}[k] \leq 0 ; \forall k \in \mathcal{T}\right\} .
$$

We will denote the EVM constraint set as $\mathcal{E}$, which can be wideband $\mathcal{E}_{\text {wb }}$ and/or frequency-selective $\mathcal{E}_{\text {fs }}$, unless stated otherwise.

Now, we rewrite the feasibility problems $(18)$ and $(19)$ as the following unconstrained problem amenable to the latter proposed efficient algorithms

$$
\underset{\overline{\boldsymbol{X}} \in \mathbb{C}^{N_{\mathrm{T}} \times N}}{\operatorname{minimize}} \mathcal{F}(\overline{\boldsymbol{X}}):=\left\{x_{\mathcal{E}}(\overline{\boldsymbol{X}})+\sum_{m=1}^{M} x_{\mathcal{C}_{m}}(\overline{\boldsymbol{X}})\right\},
$$

where the composite function $\mathcal{F}(\bar{X})$ is a sum of nondifferentiable indicator functions, i.e., $\boldsymbol{X}_{\mathcal{E}}(\cdot)$ and $x_{\mathcal{C}_{m}}(\cdot)$, of constraint sets corresponding to the wideband or frequencyselective EVM, and mask defined in (21) or (22) and 20), respectively. Hence, we seek efficient methods to solve such a problem.

Prior to developing the algorithms for the constrained EVM, we present the following theorems which are utilized for the subsequent algorithm development.

Theorem 2. If a function $f(\boldsymbol{X})=\sum_{k=1}^{n} f_{i}\left(\boldsymbol{x}_{k}\right)$ is separable across the variables column-wise $\boldsymbol{X}=\left[\boldsymbol{x}_{1}, \ldots, \boldsymbol{x}_{n}\right]$ or row-wise $\boldsymbol{X}=\left[\boldsymbol{x}_{1} ; \ldots ; \boldsymbol{x}_{N_{\mathrm{T}}}\right]$, then the respective prox operators can be shown as $\operatorname{prox}_{f}(\boldsymbol{X})=\left[\operatorname{prox}_{f_{1}}\left(\boldsymbol{x}_{1}\right), \ldots, \operatorname{prox}_{f_{n}}\left(\boldsymbol{x}_{n}\right)\right]$ or $\operatorname{prox}_{f}(\boldsymbol{X})=\left[\operatorname{prox}_{f_{1}}\left(\boldsymbol{x}_{1}\right) ; \ldots ; \operatorname{prox}_{f_{N_{\mathrm{T}}}}\left(\boldsymbol{x}_{N_{\mathrm{T}}}\right)\right]$.

Proof. Following the proximal operator Definition 3 , the minimization of the separable function is equivalent to minimization of respective functions $\left\{f_{k}\right\}$ independently [48], [49].

Theorem 3 (projection onto Frobenius norm ball [49 Lemma 6.26]). Let $E \subseteq \mathbb{C}^{p \times q}$ and $E \neq \emptyset$ be given by $E:=\mathcal{B}_{\|\cdot\|_{2}}[\boldsymbol{C}, r]=\left\{\boldsymbol{X} \in \mathbb{C}^{p \times q}:\|\boldsymbol{X}-\boldsymbol{C}\|_{F} \leq r\right\}$, then the proximal or orthogonal projection operator for the Frobenius (or $\ell_{2}$ ) norm ball, i.e., $\mathcal{B}_{\|\cdot\|_{F}}[\boldsymbol{C}, r]$ with a given center $\boldsymbol{C}$ and the radius $r$, is

$$
\begin{aligned}
\operatorname{prox}_{\lambda X_{E}}(\boldsymbol{X}) & =\operatorname{proj}_{\mathcal{B}_{\|\cdot\|_{F}[\boldsymbol{C}, r]}(\boldsymbol{X})} \\
& =\boldsymbol{C}+\left(\frac{r}{\max \left\{\|\boldsymbol{X}-\boldsymbol{C}\|_{F}, r\right\}}\right)(\boldsymbol{X}-\boldsymbol{C}) .
\end{aligned}
$$


TABLE II: Complexity comparison of various EVM-constrained and mask-compliant algorithms

\begin{tabular}{|l||c|c|}
\hline Method & Complexity: Real Multiplications & Complexity: Real Additions \\
\hline EMSP & $\mathcal{O}\left(N^{4.5} N_{\mathrm{T}}\right)$ & - \\
\hline EADMM & $\left(I(9 M N+M+6 N+2) N_{\mathrm{T}}\right)$ & $\left(I(14 M N+10 N+M-1) N_{\mathrm{T}}\right)$ \\
\hline ESSP & $\left(I\left[(5 M+7) N+1+I^{\prime}\left(4 M^{2} N^{2}+12 M N^{2}+5 M^{2} N+3 M N+M\right)\right] N_{\mathrm{T}}\right)$ & $\left(I\left[2 M N+14 N-2+I^{\prime}\left(2 M^{2} N^{2}+6 M N^{2}+6 M^{2} N-2 M N+M^{2}\right)\right] N_{\mathrm{T}}\right)$ \\
\hline
\end{tabular}

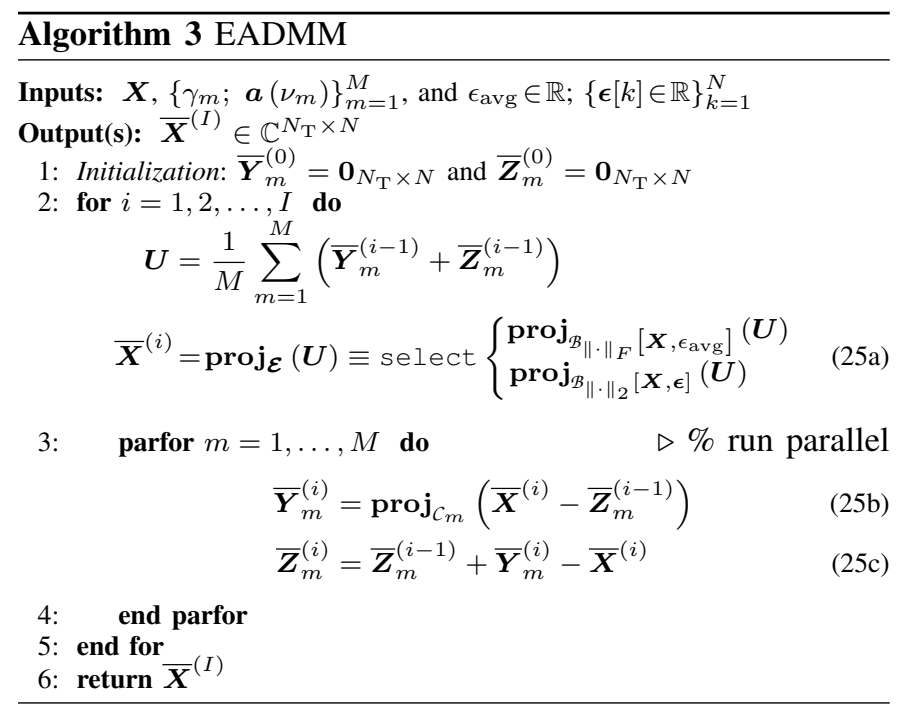

\section{B. Efficient Algorithms for EVM-Constrained LS-MSP}

In this section, we develop two computationally efficient algorithms to solve the aforementioned EVM-constrained problem 23.

1) EVM-Constrained ADMM LS-MSP solution (referred to as $E A D M M)$ : We firstly express 23 amenable to ADMM:

$$
\begin{aligned}
& \underset{\overline{\boldsymbol{X}}, \overline{\boldsymbol{Y}}_{m} \in \mathbb{C}^{N_{\mathrm{T}} \times N}}{\operatorname{minimize}} x_{\mathcal{E}}(\overline{\boldsymbol{X}})+\sum_{m=1}^{M} x_{\mathcal{C}_{m}}\left(\overline{\boldsymbol{Y}}_{m}\right) \\
& \text { subject to } \quad \overline{\boldsymbol{Y}}_{m}=\overline{\boldsymbol{X}} \quad \forall m=1, \ldots, M,
\end{aligned}
$$

The scaled-form consensus ADMM for the above problem can be expressed as [19], [20]

$$
\begin{aligned}
\overline{\boldsymbol{X}} & \leftarrow \arg \min _{\overline{\boldsymbol{X}}} x_{\mathcal{E}}(\overline{\boldsymbol{X}})+\rho \sum_{m=1}^{M}\left\|\overline{\boldsymbol{Y}}_{m}-\overline{\boldsymbol{X}}+\overline{\boldsymbol{Z}}_{m}\right\|_{F}^{2} \\
\overline{\boldsymbol{Y}}_{m} & \leftarrow \arg \min _{\overline{\boldsymbol{Y}}_{m}} x_{\mathcal{C}_{m}}\left(\overline{\boldsymbol{Y}}_{m}\right)+\rho\left\|\overline{\boldsymbol{Y}}_{m}-\overline{\boldsymbol{X}}+\overline{\boldsymbol{Z}}_{m}\right\|_{F}^{2} \forall m \\
\overline{\boldsymbol{Z}}_{m} & \leftarrow \overline{\boldsymbol{Z}}_{m}+\overline{\boldsymbol{Y}}_{m}-\overline{\boldsymbol{X}} \quad \forall m=1, \ldots, M
\end{aligned}
$$

In the first step of our proposed EADMM LS-MSP algorithm, taking the derivative with respect to $\bar{X}$ and setting to zero yields $\overline{\boldsymbol{X}}=\operatorname{prox}_{(1 / M) x_{\boldsymbol{\varepsilon}}}\left(\frac{1}{M} \sum_{m=1}^{M}\left(\overline{\boldsymbol{Y}}_{m}+\overline{\boldsymbol{Z}}_{m}\right)\right)$, i.e., the orthogonal projection onto the wideband or frequencyselective EVM constraint 25a $-\ell_{2}$ norm ball (cf. Theorem 3. The second step is an orthogonal projection onto the rank 1 quadratic constraint (cf. Theorem 1) yielding 25b. Algorithm 3 summarizes the proposed recipe for the EADMM based mask-compliant spectral precoding. The convergence analysis of the EADMM algorithm is given in Appendix A

2) EVM-Constrained SSP LS-MSP solution (referred to as ESSP): We layout the definition of Douglas-Rachford algorithm and subsequently propose the modifications to incorporate SSP algorithm for the mask constraint.

$$
\begin{aligned}
& \text { Algorithm } \mathbf{4} \text { ESSP } \\
& \text { Inputs: } \boldsymbol{X},\left\{\gamma_{m} ; \boldsymbol{a}\left(\nu_{m}\right)\right\}_{m=1}^{M} \text {, and }\left\{\boldsymbol{\epsilon} \in \mathbb{R}^{N_{\mathrm{SC}}}\right\} \text { or } \epsilon_{\mathrm{avg}} \in \mathbb{R} \\
& \text { Output(s): } \overline{\boldsymbol{X}}^{(I)} \in \mathbb{C}^{N_{\mathrm{T}} \times N} \\
& \text { 1: Initialization: } \overline{\boldsymbol{X}}_{m}^{(0)}=\boldsymbol{X} \text { and } \overline{\boldsymbol{Z}}_{m}^{(0)}=\mathbf{0}_{N_{\mathrm{T}} \times N} \\
& \text { 2: for } i=1,2, \ldots, I \text { do } \\
& \qquad \overline{\boldsymbol{Y}}^{(i)}=\operatorname{prox}_{X_{\mathcal{C}}}\left(2 \overline{\boldsymbol{X}}^{(i-1)}-\overline{\boldsymbol{Z}}^{(i-1)}\right) \quad(\text { solve SSP Alg. } 2] \\
& \qquad \overline{\boldsymbol{Z}}^{(i)}=\overline{\boldsymbol{Z}}^{(i-1)}+\lambda_{i}\left(\overline{\boldsymbol{Y}}^{(i)}-\overline{\boldsymbol{X}}^{(i-1)}\right) \\
& \qquad \overline{\boldsymbol{X}}^{(i)}=\operatorname{proj}_{\mathcal{E}}\left(\overline{\boldsymbol{Z}}^{(i)}\right) \equiv \operatorname{select}\left\{\begin{array}{l}
\operatorname{proj}_{\mathcal{B}_{\|\cdot\|_{F}}\left[\boldsymbol{X}, \epsilon_{\mathrm{avg}}\right]}\left(\overline{\boldsymbol{Z}}^{(i)}\right) \\
\operatorname{proj}_{\mathcal{B}_{\|\cdot\|_{2}}[\boldsymbol{X}, \boldsymbol{\epsilon}]}\left(\overline{\boldsymbol{Z}}^{(i)}\right)
\end{array}\right.
\end{aligned}
$$

3: end for

4: return $\overline{\boldsymbol{X}}^{(I)}$

Theorem 4 (Douglas-Rachford algorithm). Consider the following problem

$$
\underset{\overline{\boldsymbol{X}} \in \mathbb{C}^{N_{\mathrm{T}} \times N}}{\operatorname{minimize}} \mathcal{G}(\overline{\boldsymbol{X}})+\mathcal{H}(\overline{\boldsymbol{X}}),
$$

where $\mathcal{G}$ and $\mathcal{H}$ are proper closed convex functions, and which has at least one solution. Consider $\tau \in(0, \infty)$ and a sequence of relaxation parameters $\lambda_{i} \in(0,2) \quad \forall i \geq 0$ and satisfy $\sum_{i} \lambda_{i}\left(2-\lambda_{i}\right)=+\infty$ with some arbitrary initial $\bar{Z}$, then the following iterative scheme

$$
\begin{aligned}
& \overline{\boldsymbol{X}} \leftarrow \operatorname{prox}_{\tau \mathcal{G}}(\overline{\boldsymbol{Z}}) \\
& \overline{\boldsymbol{Z}} \leftarrow \overline{\boldsymbol{Z}}+\lambda_{i}\left(\operatorname{prox}_{\tau \mathcal{H}}(2 \overline{\boldsymbol{X}}-\overline{\boldsymbol{Z}})-\overline{\boldsymbol{X}}\right)
\end{aligned}
$$

converges weakly to a solution to (27).

Proof. See, e.g., [21] [52, Corollary 5.2].

Strikingly, if the problem 27) is infeasible, then for some cases one could still find an approximate solution through Douglas-Rachford method-see, e.g., [53], [54].

The Douglas-Rachford algorithm is described for two functions. However, the problem (23) at hand has more than two functions 3 . Thus, we reformulate 23 as

$$
\underset{\overline{\boldsymbol{X}} \in \mathbb{C}^{N_{\mathrm{T}}} \times N}{\operatorname{minimize}} x_{\mathcal{E}}(\overline{\boldsymbol{X}})+x_{\mathcal{C}}(\overline{\boldsymbol{X}}),
$$

where $x_{\mathcal{C}}(\overline{\boldsymbol{X}}):=\sum_{m=1}^{M} x_{\mathcal{C}_{m}}(\overline{\boldsymbol{X}})$ such that the twooperator Douglas-Rachford splitting can be employed. Since the proximal operator corresponding to the sum of $M$ indicator functions $X_{\mathcal{C}}(\overline{\boldsymbol{X}})=\sum_{m=1}^{M} X_{\mathcal{C}_{m}}(\overline{\boldsymbol{X}})$, i.e., $\operatorname{prox}_{X_{\mathcal{C}}}$ doesn't yield a closed-form, we approximate it by employing SSP. We will show numerically that ESSP framework also requires relatively less number of iterations compared to EADMM to reach desired level of performance in terms of EVM and ACLR metrics at the cost of extra computational complexity

\footnotetext{
${ }^{3}$ One could reformulate Douglas-Rachford as consensus ADMM. However, we would like to employ SSP algorithm that offers a solution to the EVMconstrained LS-MSP.
} 
TABLE III: Simulation Parameters for FDD NR (Rel-15) PDSCH Type-A

\begin{tabular}{|c|c|c|c|}
\hline Parameters & Test 1 & Test 2 & Test 3 \\
\hline Subcarrier Spacing & \multicolumn{3}{|r|}{$15 \mathrm{kHz}$} \\
\hline Carrier Bandwidth (PRB alloc.) & \multicolumn{3}{|c|}{$5 \mathrm{MHz}$ (25 PRBs) } \\
\hline Carrier Spacing for ACLR & \multicolumn{3}{|c|}{$5 \mathrm{MHz}$ upper and lower adjacent channels 5 . } \\
\hline DL SU-MIMO $N_{\mathrm{T}}, N_{\mathrm{R}}$ & $2 \mathrm{Tx}, 2 \mathrm{Rx}$ & $8 \mathrm{Tx}, 2 \mathrm{Rx}$ & $2 / 8 \mathrm{Tx}, 2 \mathrm{Rx}$ \\
\hline Spatial Layers (rank) & \multicolumn{2}{|c|}{ Fixed rank 1} & adaptive (10\% BLER) \\
\hline Spatial Precoding & \multicolumn{3}{|c|}{ (codebook-based) adaptive (10\% BLER) } \\
\hline Modulation & \multicolumn{2}{|c|}{ 64QAM } & adaptive (10\% BLER) \\
\hline Code-rate & \multicolumn{2}{|c|}{$1 / 25 / 6$} & adaptive (10\% BLER) \\
\hline Channel Model & \multicolumn{3}{|c|}{ TDL-A $(300 \mathrm{~ns}, 10 \mathrm{~Hz}) \&$ spatial correlation Low 43} \\
\hline Channel \& Noise power & \multicolumn{3}{|c|}{ Practical LMMSE based } \\
\hline HARQ max transmissions & \multicolumn{3}{|c|}{4 (3 max retransmissions with rv $\{0,2,3,1\})$} \\
\hline Other Information & \multicolumn{3}{|c|}{ LDPC; LMMSE-IRC receiver; no other impairment } \\
\hline
\end{tabular}

compared to EADMM, yet offering lower cost compared to the generic interior-point based solvers.

The proximal operator corresponding to the indicator function for EVM constraint, either wideband $\chi_{\mathcal{E}_{\mathrm{wb}}}(\overline{\boldsymbol{X}})$ or frequency-selective constraint $X_{\mathcal{E}_{\mathrm{fs}}}(\overline{\boldsymbol{X}})$, is an orthogonal projection onto Euclidean norm ball (cf. Theorem 3 ).

The ESSP framework is summarized in Algorithm 4, where we have performed a cyclic rotation of the Douglas-Rachford algorithm steps such that the proximal operator corresponding to the mask constraint occurs first in the given iteration cycle.

\section{Complexity Analysis}

In this section, we analyze the worst-case run-time complexity, in terms of required real-valued multiplications and realvalued additions but ignore the offline complexity as described in Section IV-C-see the summary in Table II

EMSP: The computational complexity of solving the optimization problems (16) and (17) -almost similar to MSP and using results in $[24]$-is $\mathcal{O}\left(N^{4.5} N_{\mathrm{T}}\right)$.

EADMM: The step (25a) is additional compared to ADMM Algorithm 1. So, the prox operator in the step 25a corresponding to the EVM constraint requires total complex multiplications $\left(I N N_{\mathrm{T}}\right),\left(I(3 N-1) N_{\mathrm{T}}\right)$, and $N+1$ real multiplications. Hence, ignoring parallelization, the total run-time complexities are $\left(I(9 M N+M+6 N+2) N_{\mathrm{T}}\right)$ and $\left(I(14 M N+10 N+M-1) N_{\mathrm{T}}\right)$ in terms of real multiplications and real additions, respectively.

ESSP: In step 26a, there are $N$ complex multiplications and $N$ real multiplications besides the computational complexity of (iterative) prox operator 26a approximated by the SSP algorithm. We need $2 N$ complex additions and $N$ real multiplications in the step 26b. Finally, in the prox operator 26c) corresponding to the EVM constraint same as in EADMM need to be considered. Therefore, the total online complexities in terms of real multiplications and additions are given in Table II where $I^{\prime}$ corresponds to inner iterations using SSP Algorithm 2

\section{Vi. Performance Evaluation}

In this section, we evaluate the performance of the proposed algorithms for mask-compliant spectral precoding that are both EVM-unconstrained and EVM-constrained utilizing a 5G NR (Rel-15) compliant inhouse link-level simulator. Moreover, we compare the performance of the proposed algorithms with the conventional spectral precoding algorithms, accordingly.
TABLE IV: EVM Requirements 5

\begin{tabular}{|l|c|}
\hline Modulation Scheme & EVM Threshold \\
\hline QPSK & $17.5 \%$ \\
\hline 16QAM & $12.5 \%$ \\
\hline 64QAM & $8.0 \%$ \\
\hline
\end{tabular}

\section{A. Performance Measures}

We analyze the spectral precoding performance in terms of two figure-of-merits, namely OOBE and in-band distortions, in particular assuming base station supporting sub- $6 \mathrm{GHz}$, e.g. frequency ranges between $410 \mathrm{MHz}$ and $7.125 \mathrm{GHz}$-referred to as FR1 in 5G NR [5, Section 5.1].

1) Out-of-Band Distortion: As mentioned in Section III-B1, mask and (conducted) ACLR are typically the performance metrics to quantify the operating band unwanted emissions.

In practical systems, the (digital) spectrum shaping is followed by other (non-linear) digital and analog processing as illustrated in Fig. 11. Consequently, there is some spectral regrowth phenomenon due to such (non-linear) components in the transmitter after spectrum shaping. Thus, an implementation margin in terms of ACLR and mask requirements are necessary to cope with spectral regrowth. Hence, OOBE performance must render better performance than the (overall) stipulated mask in the standard due to spectrum shaping to account for the margin.

We have considered ACLR corresponding to the 1st adjacent carrier in both upper and lower frequencies, where the minimum requirement is $45 \mathrm{~dB}$-worst-case of measured ACLR in the upper and lower channels [5, Section 6.6.3]. It is worth highlighting that these ACLR requirements are for the complete radio chain, i.e., measurements need to be performed at the antenna connector. Thus, spectrum shaping may have some aggressive mask and ACLR requirements to meet the minimum requirements at the antenna connector.

2) In-Band Distortion: In these simulations, the in-band distortion is not only quantified in terms of EVM but also in terms of BLER [56] and throughput [57].

For our link simulations, we present normalized throughput, i.e., normalizing the throughput results by the maximum achievable throughput without any spectral precoding or OOBE reduction and other hardware impairments or imperfections.

\section{B. Simulation Parameters and Assumptions}

The key simulation parameters for the physical downlink shared channel (PDSCH) with type- $\mathrm{A}^{4}$ and the three investigated test scenarios are summarized in Table III] see, e.g., [5], [58], for the detailed NR physical layer and performance requirements. We have considered $15 \mathrm{kHz}$ subcarrier spacing for the NR numerology unless otherwise mentioned. Furthermore, no supporting signals are transmitted besides PDSCH along with the demodulation reference signal (DMRS) for the practical channel and noise variance estimation at the UE side. Note that for simulations purpose, we have considered relatively narrow $5 \mathrm{MHz}$ channel bandwidth with 25 physical

\footnotetext{
${ }^{4}$ These data types refer to different PDSCH demodulation reference signals allocation [44].
} 


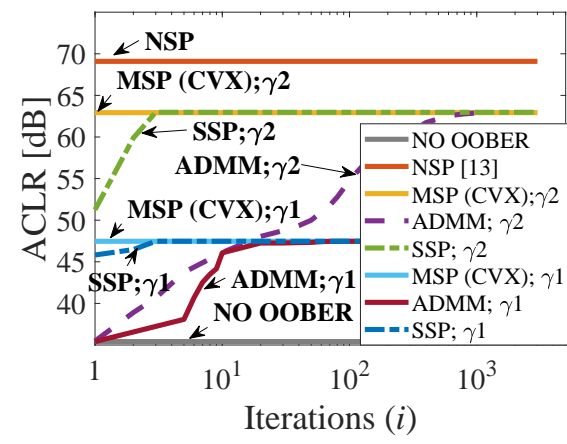

(a) ACLR-vs.-Iterations.

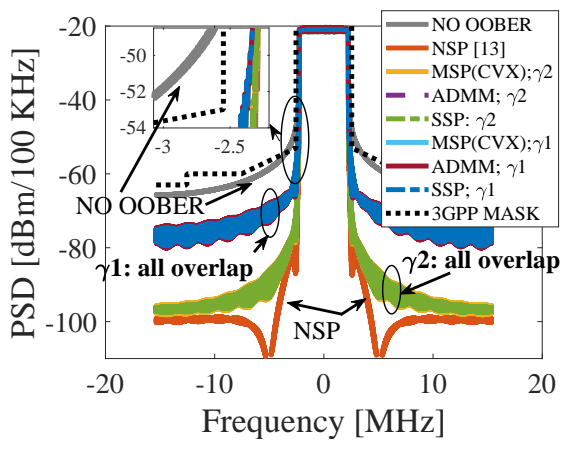

(b) PSD at the final iteration.

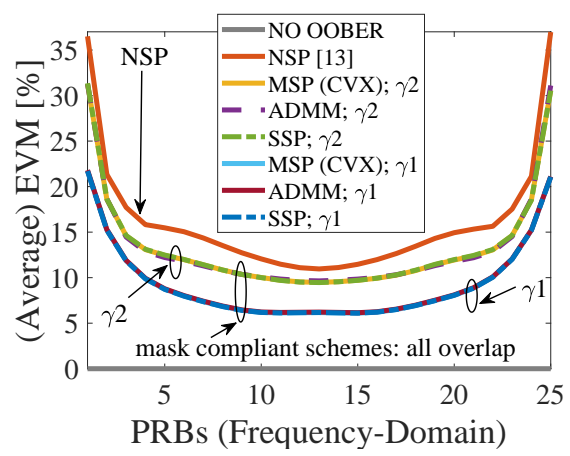

(c) EVM $[\%]$ at the final iteration.

Fig. 2: (a) Convergence behaviour of EVM-unconstrained algorithms considering two different target masks, while (b) and (c) are achieved PSD and EVM at the final iteration.

resource block (PRB)s allocation, even though the proposed methods can be employed for arbitrary bandwidths. Furthermore, we have employed a low spatial correlation model, but the complexities of the proposed algorithms are oblivious of spatial correlation-cf. Table I] and II However, the inband performance may degrade with increasing correlation, notably for high spatial rank setup. In Table IV] we have provided 3GPP NR wideband average EVM 5 requirements [5] as a reference according to the considered modulation alphabet.

In addition to the parameters given in Table III] the discrete frequencies for mask compliant precoders are selected as $\nu \in\{\mp 5010, \mp 4995, \mp 2565, \mp 2550\} \mathrm{kHz}$, where the negative and positive frequencies correspond to the left and right side of the out-of-band (OOB) of the occupied signal spectrum, respectively. Notice that these discrete frequencies can be asymmetrically selected for the OOBE suppression. The considered mask, referred to as mask-1 is $\gamma_{\text {mask }-1}:=\gamma 1=$ $[-75,-75,-65,-65] \mathrm{dBm} / 100 \mathrm{kHz}$, corresponding to left/right side of the signal spectrum. Furthermore, we have considered another aggressive target mask-2, i.e., $\gamma_{\text {mask }-2}:=\gamma 2=$ $[-85,-85,-75,-75] \mathrm{dBm} / 100 \mathrm{kHz}$ for ACLR and EVM performance, particularly. For this work, we have not optimized the selection of the discrete frequencies' set for the precoding.

Based on our numerical grid-search utilizing Test 3 with 2 transmit antennas (cf. Table IIII), we found a suitable $\rho=10$ for consensus ADMM algorithm (cf. Algorithm 11). For the MSP (8) solution, we have employed CVX wrapper with SDPT3 solver [47]. Note that we do not have any additional radio hardware impairments at the transmitter and/or receiver besides the distortion generated at the transmitter by the considered spectral precoders, if enabled.

Furthermore, for benchmarking purpose, we have also evaluated notching spectral precoder (NSP) [13] and EVMconstrained NSP (ENSP) [26], where for each transmit antenna branch NSP and ENSP are performed independently. More specifically, for each $j$-th transmit antenna, we perform $\overline{\boldsymbol{d}}_{j}=\boldsymbol{G} \boldsymbol{d}_{j}$, where $\boldsymbol{G}=\boldsymbol{I}_{N}-\alpha \boldsymbol{A}^{\mathrm{H}}\left(\boldsymbol{A} \boldsymbol{A}^{\mathrm{H}}\right)^{-1} \boldsymbol{A}$ and $\boldsymbol{A}[m, k]$ in

\footnotetext{
${ }^{5}$ The NR standard puts a requirement on the equalized EVM, cf. $\sqrt{5}$ Section 6.5.2.2], e.g., for 64QAM, the transmitter is allowed to induce EVM up to $8 \%$. As mentioned in footnote 1 (Page 4), the unequalized EVM is an upper bound for the equalized EVM, i.e., unequalized EVM is tougher requirement than equalized, see, e.g., [46].
}

(6). For NSP $\alpha=1$, and ENSP $\alpha \in \mathbb{R}$ is a tunable parameter to meet the desired EVM.

\section{Simulation Results}

In this section, we present simulation results for both unconstrained and constrained EVM spectral precoding methods.

In Fig. 2, we present the convergence behaviour of the proposed EVM-unconstrained algorithms in terms of OOB and in-band performance considering Test 3 (cf. Table III) and two different target masks, i.e., mask-1 and mask-2.

Figure 2|a) shows the ACLR performance against iterations. Evidently, SSP converges in 2-3 iterations for both target masks to achieve the same ACLR results as rendered by MSP(CVX). Under relaxed mask-1 constraint, ADMM requires nearly 80 iterations, while for aggressive mask-2 it requires approximately 800 iterations. On the other hand, we have observed that CVX achieves the MSP solution in nearly 25 iterations, but we could not manage to extract the result for every single iteration; hence, we show the final result rendered by the CVX solver corresponding to the last iteration in the figures. We have also observed similar behaviour for EVM against iterations. For the subsequent EVM-unconstrained results, we consider mask- 1 and fix the iterations of ADMM and SSP as 80, and 2, respectively. Furthermore, we have numerically observed that these spectral precoders have a negligible impact on the peak to average power ratio.

Figure 2|(b) exhibits the average PSD versus frequency for the proposed algorithms and both masks considering final iterations of the respective algorithms. The NR mask corresponding to a medium range $\mathrm{BS}$ with the maximum output of $38 \mathrm{dBm}$ [5, Section 6.6.4] is also shown for the completeness, but the mask is normalized according to the normalized transmit signal power of $0 \mathrm{dBm}$, i.e., approximately -21.5 $\mathrm{dBm} / 100 \mathrm{kHz}$, in the link simulations. All the proposed methods in addition to the prior art fulfils the 3GPP NR mask.

Figure 2 $2(\mathrm{c})$ depicts the average EVM distribution per PRBs [58]. The edge PRBs have relatively high distortion power compared to the central PRBs. Notice that the NR bandwidth is slightly asymmetric with respect to the direct-current carrier. Moreover, the discrete selected frequency points $(\boldsymbol{\nu})$ are not symmetric with respect to the direct-current carrier. Therefore, one could consequently observe that the EVM distribution in the frequency-domain is asymmetric. 


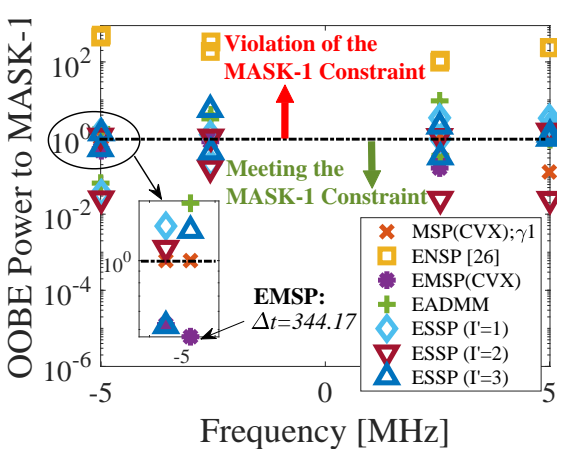

(a) Ratio of OOBE power to mask-1.

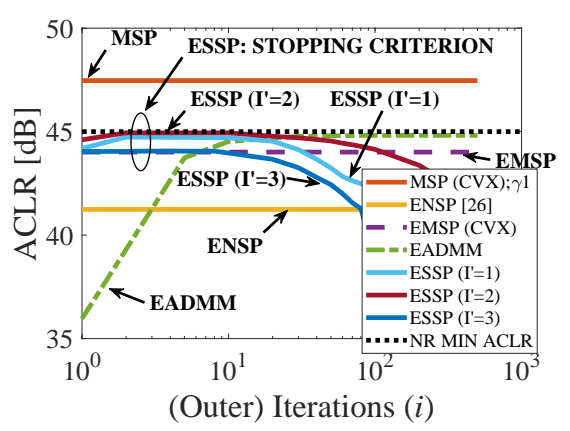

(b) ACLR-vs.-Iterations of ESSP.

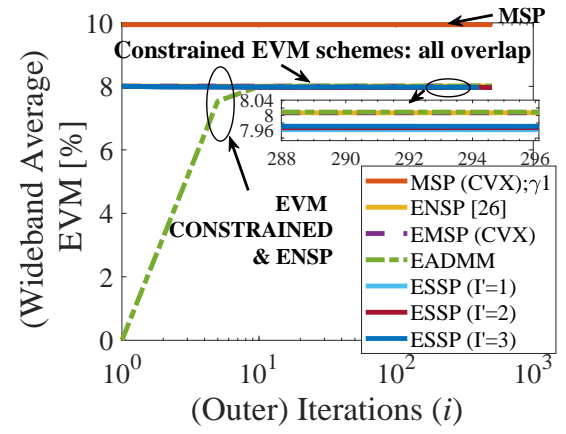

(c) EVM-vs.-Iterations.

Fig. 3: Convergence behaviour of wideband $8 \%$ EVM-constrained spectral precoding.

Subsequently, we present the performance of EVMconstrained while considering mask-1 constraint. In Fig. 3, we exhibit the convergence behaviour of the proposed wideband EVM-constrained algorithms in terms of the in-band, namely, EVM, and OOB performance, namely, ACLR and PSD. For these simulations, we have considered $8 \%$ wideband unequalized EVM.

Figure $3($ a) illustrates that the problem $(23)$ is infeasible, i.e., the intersection of the two convex sets corresponding to mask and EVM constraints are empty, by considering a single OFDM symbol and by randomly selecting any of the transmit antenna branch corresponding to Test 3 . More specifically, we show OOBE power to the target mask, i.e., $\left(\left|\boldsymbol{A} \overline{\boldsymbol{d}}_{j}\right|^{2}\right) \oslash(\Delta t \gamma)$, where $\oslash$ denotes elementwise division. If this ratio is larger than 1 , then it implies that the target mask constraint is violated at the considered $\nu$-th discrete frequency point. On the contrast, if this ratio is less than and equal to 1 , the mask constraint is met by the spectrally precoded OFDM symbol. Notice that, in the case of EMSP, $\Delta t=344.17$ was obtained from CVX [47] for the given OFDM symbol and transmit antenna branch, which implies that the original problem is infeasible. As it can be observed from the figure, the solution rendered by the MSP naturally meets the mask constraint since it is an inequality constrained minimization problem, i.e., this ratio will always be less than or equal to 1 as it is a feasible problem. The spectrally precoded symbol generated by ENSP algorithm does not meet any of the 8 mask constraints while the EVM constraint is kept to $8 \%$. The symbol generated by EMSP meets the target mask constraints but after considering $\Delta t=344.17$, which evidently penalizes the achieved ACLR compared to the ACLR achieved through MSP algorithm in order to meet the wideband $8 \%$ EVM constraint. Furthermore, the precoded symbol rendered by EADMM and ESSP apparently show that for some discrete frequency points mask constraint is met while for some points it fails. Therefore, long-term average of PSDs or ACLRs over several slots of OFDM signals generated by the proposed algorithms outperform significantly heuristic ENSP method in terms of out-of-band performance. In other words, we can construe from the figure that the EVM constraint penalizes the achievable ACLR compared to the ACLR achieved by unconstrained EVM problem.

Figure $3(\mathrm{~b})$ demonstrate the ACLR performance against iterations for all the proposed EVM-constrained spectral pre- coding algorithms. In the case of ESSP, we illustrate the performance with three different internal iterations corresponding to the prox operator for the sum of the indicator functions to the mask constraints, i.e., calling SSP algorithm. Strikingly, the ACLR performance accomplished by ESSP without stopping criterion diverges after typically 2 iterations, although the wideband EVM performance convergence behaviour is consistent over the iterations, i.e., meeting the wideband EVM constraint—see Fig. $3(\mathbf{c})$. Remarkably, the result rendered by EADMM do not digress or diverge over iterations, unlike observed in Douglas-Rachford-based ESSP algorithm without early stopping criterion. Thus, ESSP requires a stopping criterion to render an approximate solution. We choose to terminate the outer iterations within ESSP when the ACLR performance degrades with the increasing iterations. Hence, we employ an early stopping criterion for the results presented in the sequel, which typically terminates the algorithm at around 2 outer iterations (for all the considered test cases).

Figure 4 demonstrates the convergence behaviour of frequency-selective EVM algorithms. The frequency-selective EVM constraints are arbitrarily chosen to exhibit the efficacy of the proposed frequency-selective algorithms. The first lower and upper edge PRBs were set to have EVM requirement $[20 \%, 20 \%, 19 \%, 19 \%, 16 \%, 15 \%, 14 \%, 13 \%, 12.5 \%, 12.5 \%$, $12.5 \%, 12.5 \%]$ such that the edge most subcarrier of the edge PRB corresponds to $20 \%$ EVM and the inner most subcarrier corresponds to $12.5 \%$ EVM. Furthermore, all the subcarriers of the second, third, and fourth edge PRBs are set to have the flat target of $12 \%, 9.5 \%$, and $8 \%$ EVM, respectively. The remaining central subcarriers are set to $7 \%$. This represents a wideband average EVM of $\sim 8.9 \%$ across the subcarriers. The EVM distribution over subcarriers are shown in Fig. 4(a) where all the proposed methods meet the frequency-selective EVM requirement. In Fig. $4(\mathrm{c})$, all the frequency-selective EVM algorithms have similar wideband EVM performance. The imposed mask and frequency-selective EVM constraints make the problem infeasible which is evident from the divergence of ACLR metric rendered by ESSP algorithms without any stopping criterion as depicted in Fig. 4(b) We observe that the ACLR performance delivered by EADMM are quite stable over iterations even though the problem is infeasible. Reiterating that the ENSP can not be employed or extended to support frequency-selective EVM requirement.

In Fig. 5. we exhibit in-band and OOBE performance 


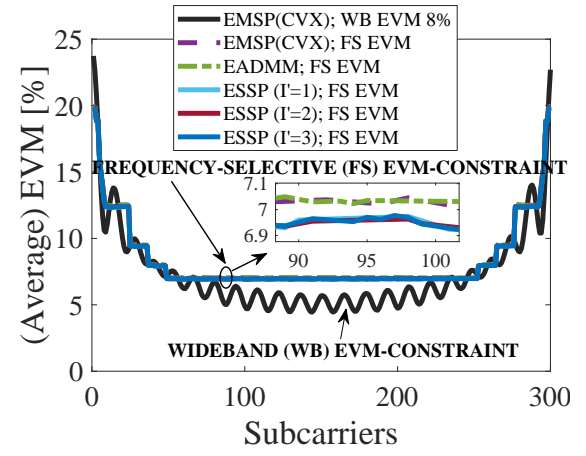

(a) EVM [\%] over subcarriers.

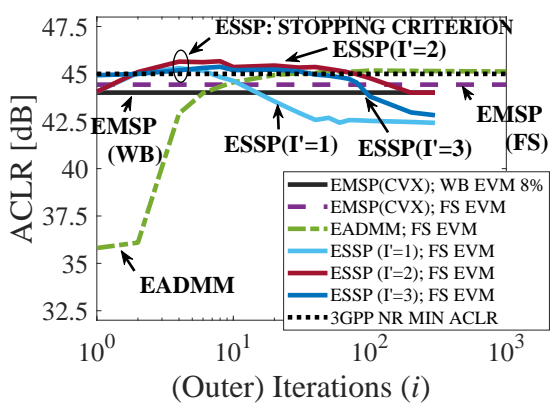

(b) ACLR-vs.-Iterations of ESSP.

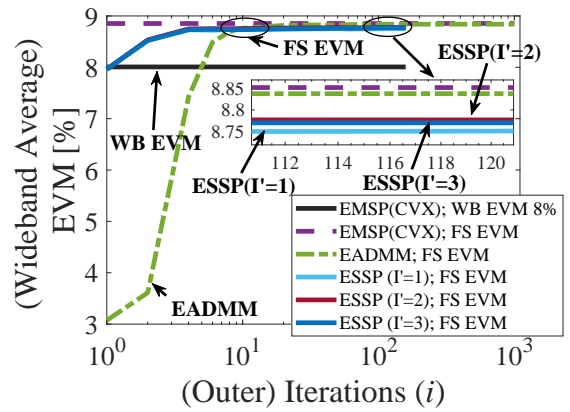

(c) EVM-vs.-iterations.

Fig. 4: Convergence behaviour of frequency-selective EVM-constrained spectral precoding.

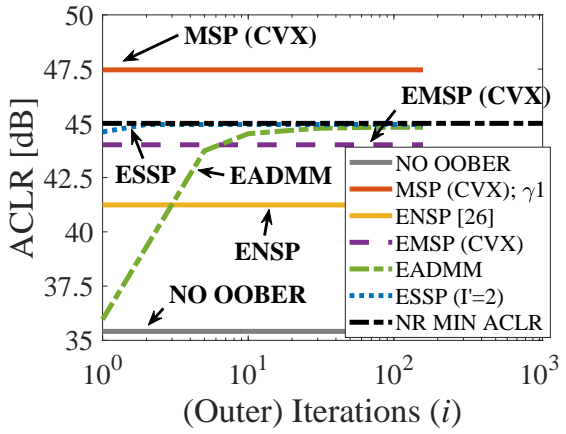

(a) ACLR-vs.-Iterations for Test 3 $(8 \mathrm{~T} x 2 \mathrm{Rx})$.

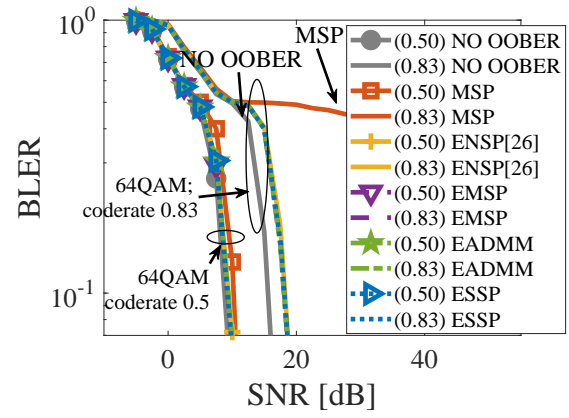

(d) BLER-vs.-SNR for Test 1 (2Tx2Rx, Rank 1).

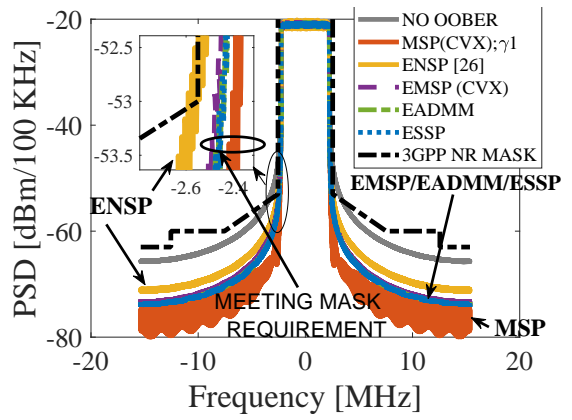

(b) PSD for Test $3(8 \mathrm{~T} x 2 \mathrm{Rx})$.

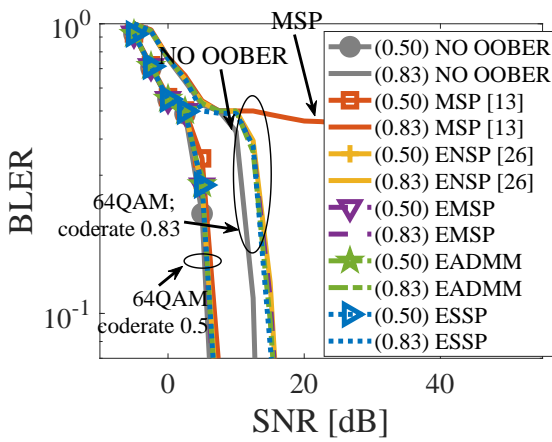

(e) BLER-vs.-SNR for Test 2 (8Tx2Rx, Rank 1).

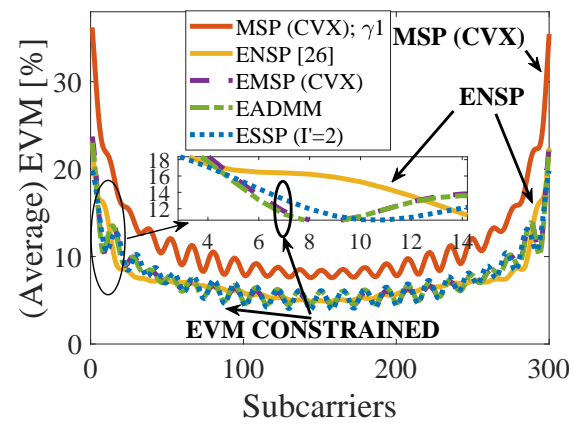

(c) EVM distribution for Test $3(8 \mathrm{~T} x 2 \mathrm{Rx})$.

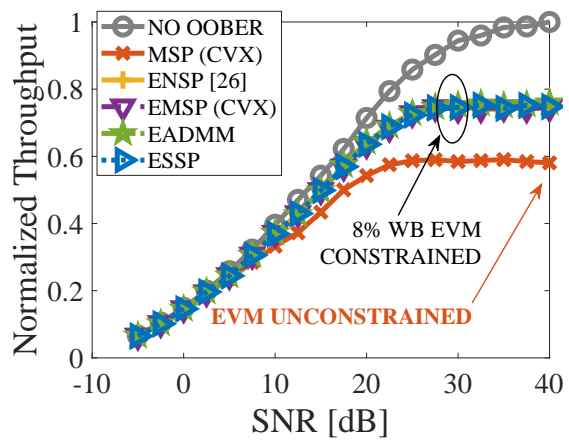

(f) Throughput-vs.-SNR for Test 3 (8Tx2Rx).

Fig. 5: Performance of wideband $8 \%$ EVM-constrained spectral precoding including ESSP with stopping criterion.

of the proposed $8 \%$ wideband EVM-constrained algorithms, where ESSP employs 2 inner and 2 outer iterations (with stopping criterion) for all the 3 test cases. Further, EADMM employs 40 iterations for the performance evaluation. More specifically, we show the in-band performance, in terms of BLER and (normalized) throughput against received SNR at the user-equipment and EVM distribution for the final chosen iterations of the respective algorithms. We also depict the OOBE performance, in terms of ACLR versus (outer) iterations and average PSDs of the the final chosen iterations of the respective algorithms.

Particularly, Figure 5(a) depicts ACLR performance against iterations utilizing Test 3 (similar results are observed for other test scenarios). Since the problem was infeasible, the EVM constraint penalizes the achievable ACLR compared to the ACLR achieved by unconstrained EVM problem, which can be corroborated by the EMSP performance comparing with the performance achieved by the MSP (CVX). Moreover, it can be noticed that all the algorithms have different ACLR performance and unfortunately do not meet the minimum $45 \mathrm{~dB}$ ACLR requirement for the 1st channel. Still, all the proposed algorithms render the ACLR between $44 \mathrm{~dB}$ and $45 \mathrm{~dB}$. Conspicuously, ESSP achieves $44.95 \mathrm{~dB}$ ACLR, which is 0.05 $\mathrm{dB}$ below the minimum $45 \mathrm{~dB}$ requirement. Moreover, it can be observed that all the proposed algorithms meet the 3GPP mask requirement as illustrated in the PSD-see Fig. 5)(b) Therefore, one could employ a relaxed additional spectrum shaping method, for instance, a transmit windowing or filtering that would use less than $9 \%$ of the cyclic prefix length to meet the ACLR requirement with some implementation margin. In other words, one can combine spectral precoding with other (time-domain) spectrum shaping methods, see, e.g., [18] 
and thereby improve OOBE performance. For completeness, Fig. 5F(c) depicts the distribution of EVM over subcarriers (in frequency-domain) after the final iterations of the respective algorithms.

In Fig. 5(a) the ACLR performance rendered by the heuristic ENSP method is, inadequately low, $41.24 \mathrm{~dB}$ compared to the proposed principled methods that are between $44 \mathrm{~dB}$ and $45 \mathrm{~dB}$, i.e., ENSP has $3-4 \mathrm{~dB}$ loss in ACLR but similar wideband EVM. Furthermore, ENSP fails to meet the mask requirement, cf. Fig. 55(b). Thus, ENSP would need an aggressive additional spectrum shaping method, for instance transmit windowing with nearly more than $30 \%-40 \%$ cyclic prefix length, to meet both mask and ACLR requirement. Hence, the gain from such spectral precoding vanishes and may be futile to employ in a realistic system.

In Fig. 5, we also manifest the in-band performance of the proposed algorithms considering wideband $8 \%$ EVM constraint. In particular, we show BLER against received SNR at the user-equipment for the Test 1 and Test 2 since there is no link adaptation in these tests except for the spatial precoding matrix indicator (PMI) adaptation, where all the proposed EVM-constrained algorithms have similar performance. Furthermore, we present normalized throughput versus received SNR for the Test 3 with 8 transmit antennas since this test employs fast link adaptation, i.e., spatial precoder (PMI), rank/layers, and modulation and code rate adaptation (referred to as channel quality indicator in the 3GPP standard) with $10 \%$ target BLER on long-term average. From these figures, firstly, we observe that the distribution of the EVM in the frequency domain matters since meeting minimum $8 \%$ wideband EVM requirement for 64QAM may not be sufficient to meet the receiver demodulation performance. Secondly, we observe that increasing number of the transmit antennas from 2 to 8 for the low-rank scenario does not improve the in-band performance gain. We have observed even with 8 transmit antennas that the EVM emanating from spectral precoding is beamformed in the same direction as the signal, similar observations for (large scale) MIMO for the EVM stemming from other sources, see, e.g., [28]-[31]. The EVM-constrained algorithms improve the receiver demodulation performance compared to the EVMunconstrained spectral precoding. However, there is a potential to improve receiver performance while meeting the OOBE requirements when the transmitter can be aware of the full channel state information of the receiver(s), which will be addressed in future work.

\section{CONCLUSION}

In this paper we investigated the problem of spectral precoding with mask compliant properties. The problem is formally posed as some optimizations that offer an explicit trade-off between EVM and OOBE suppression for large-scale MIMOOFDM systems, but have the disadvantage of not having closed-form solutions. To mitigate the complexity of such problems, we proposed a divide-and-conquer approach that decomposes the spectral precoding problem into smaller problems having each either a closed-form or an efficient solution. More specifically, in the first part of the paper, we developed two computationally efficient algorithms for the EVM- unconstrained spectral precoding method, namely 1) ADMM and 2) SSP-which is derived by utilizing KKT conditions, capitalizing on the closed-form solution of rank 1 quadratic form, and a coordinate descent scheme. In the second part-in stark contrast to the unsystematic prior art on wideband EVMconstrained spectral precoding-we formulated the optimal wideband and frequency-selective constrained EVM problems in conjunction with mask-compliant spectral precoding. Subsequently, employing the divide-and-conquer approach, we extended and developed "hardware-friendly" algorithms for the EVM-constrained spectral precoding method, referred to as 1) EADMM and 2) ESSP — which uses the Douglas-Rachford operator splitting technique to meet an EVM constraint while internally utilizes SSP for mask constraint. The proposed algorithms should be treated as vendor-specific transmitter module like filtering, which implies that they are 3GPP NR standard transparent to the transmitter and the receiver. Numerical results corroborate that the proposed low-complexity algorithms can meet the target EVM constraints and the 3GPP NR mask by suppressing OOBE.

This is arguably the first work that proposes computationally affordable EVM-constrained and yet mask-compliant spectral precoding.

\section{APPENDIX A}

CONVERgence AnAlysis: ADMM-Algorithm 1 And EADMM-ALGORITHM 3

We present the convergence of Algorithm 1 and Algorithm 3 in a consolidated manner.

Theorem 5 (Global convergence of consensus ADMM or EADMM). Consider an either EVM unconstrained (9) or constrained problem (23) that can be unified as, i.e.,

$$
\begin{array}{cl}
\underset{\left\{\overline{\boldsymbol{Y}}_{m} \in \mathbb{C}^{N_{\mathrm{T}} \times N}\right\}, \overline{\boldsymbol{X}} \in \mathbb{C}^{N_{\mathrm{T}} \times N}}{\text { minimize }} & \sum_{m=1}^{M} f_{m}\left(\overline{\boldsymbol{Y}}_{m}\right)+g(\overline{\boldsymbol{X}}) \\
\text { subject to } & \overline{\boldsymbol{Y}}_{m}=\overline{\boldsymbol{X}},
\end{array}
$$

where non-differentiable indicator function $f_{m}:=x_{\mathcal{C}_{m}}$ to the mask constraint set (see (20)) for all $m=1, \ldots, M$ is closed convex proper (common to both ADMM and EADMM). The closed convex proper function is either differentiable $g:=\|\bar{X}-\boldsymbol{X}\|_{F}(E V M$ unconstrained $)$ or non-differentiable indicator function $g:=X_{\mathcal{E}}$ (for either wideband (21) or frequency-selective (22) EVM constraint). Suppose (30) has at least one solution. Now, assume subproblems of ADMM and EADMM have solutions, and so-called dual residual $\lim _{i \rightarrow+\infty}\left(\overline{\boldsymbol{X}}^{(i+1)}-\overline{\boldsymbol{X}}^{(i)}\right)=0$ and primal residual $\lim _{i \rightarrow+\infty}$ $\left(\overline{\boldsymbol{Y}}_{m}^{(i+1)}-\overline{\boldsymbol{X}}^{(i+1)}\right)=0, \forall m=1, \ldots, M$, and $\rho \in \mathbb{R}_{>0}$ with some arbitrary initial $\left\{\overline{\boldsymbol{X}}^{(0)}, \overline{\boldsymbol{Y}}_{m}^{(0)}, \overline{\boldsymbol{Z}}_{m}^{(0)}\right\}$. Then, Algorithm 1 and Algorithm 3, at any limit point, $\left\{\overline{\boldsymbol{X}}^{(i)}\right\}$ converge to a KKT point of 30 .

Proof. Towards the convergence analysis goal of the proposed ADMM and EADMM algorithms, we follow the proof given in, e.g., [20]. Now, we form the (unaugmented) Lagrangian of the unified problem (30) proposed to be solved by ADMM/EADMM such that 


$$
\begin{aligned}
& \mathcal{L}\left(\left\{\overline{\boldsymbol{Y}}_{m}\right\}_{m=1}^{M}, \overline{\boldsymbol{X}},\left\{\overline{\boldsymbol{Z}}_{m}\right\}_{m=1}^{M}\right) \\
& :=\sum_{m=1}^{M} f_{m}\left(\overline{\boldsymbol{Y}}_{m}\right)+g(\overline{\boldsymbol{X}})+\sum_{m=1}^{M} 2 \Re\left\{\operatorname{Tr}\left(\overline{\boldsymbol{Z}}_{m}^{\mathrm{H}}\left(\overline{\boldsymbol{Y}}_{m}-\overline{\boldsymbol{X}}\right)\right)\right\},
\end{aligned}
$$

where $\operatorname{Tr}$ is a trace operator. Using (31), then according to KKT optimality conditions-see, e.g., [25], in particular, stationarity condition at the optimal primal values $\left\{\overline{\boldsymbol{Y}}_{m}^{\star}\right\}_{m=1}^{M}$ and $\overline{\boldsymbol{X}}^{\star}$, and dual variable $\left\{\overline{\boldsymbol{Z}}_{m}^{\star}\right\}_{m=1}^{M}$ satisfy

$$
\begin{aligned}
& \mathbf{0} \in \frac{\partial}{\partial\left(\overline{\boldsymbol{X}}^{\star}\right)^{*}} \mathcal{L}\left(\left\{\overline{\boldsymbol{Y}}_{m}^{\star}\right\}_{m=1}^{M}, \overline{\boldsymbol{X}}^{\star},\left\{\overline{\boldsymbol{Z}}_{m}^{\star}\right\}_{m=1}^{M}\right) \\
& \Longleftrightarrow \mathbf{0} \in \partial g\left(\overline{\boldsymbol{X}}^{\star}\right)-\sum_{m=1}^{M} \overline{\boldsymbol{Z}}_{m}^{\star} \\
& \quad \mathbf{0} \in \frac{\partial}{\partial\left(\overline{\boldsymbol{Y}}_{m}^{\star}\right)^{*}} \mathcal{L}\left(\left\{\overline{\boldsymbol{Y}}_{m}^{\star}\right\}_{m=1}^{M}, \overline{\boldsymbol{X}}^{\star},\left\{\overline{\boldsymbol{Z}}_{m}^{\star}\right\}_{m=1}^{M}\right) \\
& \Longleftrightarrow \mathbf{0} \in \partial f_{m}\left(\overline{\boldsymbol{Y}}_{m}^{\star}\right)+\overline{\boldsymbol{Z}}_{m}^{\star},
\end{aligned}
$$

where, for EVM unconstrained, $\partial g\left(\overline{\boldsymbol{X}}^{\star}\right)=\left\{\left(\overline{\boldsymbol{X}}^{\star}-\boldsymbol{X}\right)\right\}$ and, for EVM constrained, $\partial g\left(\overline{\boldsymbol{X}}^{\star}\right)=\partial x_{\mathcal{E}} \equiv \mathcal{N} \mathcal{E}$, where $\mathcal{N}_{\mathcal{E}}$ corresponds to a normal cone (see, e.g., [49, Chapter 3]); and $\partial f_{m}\left(\overline{\boldsymbol{Y}}_{m}^{\star}\right)=\partial x_{\mathcal{C}_{m}} \equiv \mathcal{N}_{\boldsymbol{C}_{m}}$. The primal feasibility satisfies

$$
\overline{\boldsymbol{Y}}_{m}^{\star}-\overline{\boldsymbol{X}}^{\star}=\mathbf{0} \quad \forall m=1, \ldots, M .
$$

Towards this end, we analyze the proposed Algorithm 1 and Algorithm 3, which for sufficiently large iterations satisfy the abovementioned optimality conditions using the stated assumptions. In the first step of (E)ADMM, $\overline{\boldsymbol{X}}^{(i+1)}$ minimizes the update step-1 (cf. 24a) $)$, i.e., $\quad 0 \in \partial g\left(\overline{\boldsymbol{X}}^{(i+1)}\right)$ $\sum_{m=1}^{M} \rho\left(\overline{\boldsymbol{Y}}_{m}^{(i)}-\overline{\boldsymbol{X}}^{(i+1)}+\overline{\boldsymbol{Z}}_{m}^{(i)}\right)$. Using dual variable update 24c and rearranging the terms yields $\mathbf{0} \in \partial g\left(\overline{\boldsymbol{X}}^{(i+1)}\right)$ $\sum_{m=1}^{M} \rho \overline{\boldsymbol{Z}}_{m}^{(i+1)}+\sum_{m=1}^{M} \rho\left(\overline{\boldsymbol{Y}}_{m}^{(i+1)}-\overline{\boldsymbol{Y}}_{m}^{(i)}\right)$. Now, we state $\left(\overline{\boldsymbol{Y}}_{m}^{(i+1)}-\overline{\boldsymbol{Y}}_{m}^{(i)}\right) \rightarrow 0$ when $i \rightarrow \infty$ because of the assumption that the dual residual $\left(\overline{\boldsymbol{X}}^{(i+1)}-\overline{\boldsymbol{X}}^{(i)}\right) \rightarrow 0$ and the primal residual $\left(\overline{\boldsymbol{Y}}_{m}^{(i+1)}-\overline{\boldsymbol{X}}^{(i+1)}\right) \rightarrow 0$. Thus, asymptotically, $\overline{\boldsymbol{X}}^{(i+1)}$ update satisfies the stationarity condition (32). Similarly, we have $\mathbf{0} \in \partial f_{m}\left(\overline{\boldsymbol{Y}}_{m}^{(i+1)}\right)+\rho\left(\overline{\boldsymbol{Y}}_{m}^{(i+1)}-\overline{\boldsymbol{X}}^{(2+1)}+\overline{\boldsymbol{Z}}_{m}^{(i)}\right)=$ $\partial f_{m}\left(\overline{\boldsymbol{Y}}_{m}^{(i+1)}\right)+\rho \overline{\boldsymbol{Z}}_{m}^{(i+1)}$ in the step-2 update (cf. $\left.24 \mathrm{~b}\right)$ ), where in the last equality have used the dual variable update 24c . Thus, step-2 always satisfies the stationarity condition (33) for sufficiently large $i$.

Finally, primal feasibility (34) is satisfied by the assumption $\left(\overline{\boldsymbol{Y}}_{m}^{(i+1)}-\overline{\boldsymbol{X}}^{(i+1)}\right)=0$, when $i \rightarrow+\infty$.

\section{APPENDIX B}

PROOF OF (13): PROJECTION ONTO RANK 1 ELLIPSOID

The projection problem reads $\underset{z}{\operatorname{minimize}}\|\boldsymbol{x}-\boldsymbol{z}\|_{2}^{2}$ subject to $\boldsymbol{z}^{\mathrm{H}} \widetilde{\boldsymbol{A}} \boldsymbol{z}-b \leq 0$ where $\widetilde{\boldsymbol{A}}=\boldsymbol{u} \boldsymbol{u}^{\mathrm{H}}$. So, the Lagrangian can be formed as $L(\boldsymbol{z}, \mu)=\|\boldsymbol{x}-\boldsymbol{z}\|_{2}^{2}+\mu\left(\left|\boldsymbol{u}^{\mathrm{H}} \boldsymbol{z}\right|^{2}-b\right)$. According to the KKT conditions [25], in particular due to complementary slackness condition, if the given $\boldsymbol{x}$ is feasible, that is, fulfils the constraint then $\boldsymbol{z}=\boldsymbol{x}$ and the Lagrange multiplier would correspond to $\mu=0$. However, if the given $\boldsymbol{x}$ does not fulfil the constraint, then $\mu>0$ and the inequality constraint can be converted to the equality constraint such that constraint $\left|\boldsymbol{u}^{\mathrm{H}} \boldsymbol{z}\right|^{2}=b \Longleftrightarrow \boldsymbol{u}^{\mathrm{H}} \boldsymbol{z}=\sqrt{b} \exp (\iota \theta)$ for some unknown angle $\theta$. Now, we assume that the angle $\theta$ is known, then the Lagrangian can be expressed as $L\left(\boldsymbol{z}, \mu^{\prime}\right)=\|\boldsymbol{x}-\boldsymbol{z}\|_{2}^{2}+\mu^{\prime}\left(\boldsymbol{u}^{\mathrm{H}} \boldsymbol{z}-\sqrt{b} \exp (\iota \theta)\right)$.

Now, according to the KKT conditions, we set gradient of $L\left(\boldsymbol{z}, \mu^{\prime}\right)$ with respect to $\boldsymbol{z}$ to 0 , such that $\boldsymbol{z}=\boldsymbol{x}-\mu^{\prime} \boldsymbol{u}$. Plugging this in the constraint yields $\mu^{\prime}=\frac{\boldsymbol{u}^{\mathrm{H}} \boldsymbol{x}-\sqrt{b} \exp (\iota \theta)}{\|\boldsymbol{u}\|_{2}^{2}}$. Thus, $\boldsymbol{z}=\boldsymbol{x}-\mu^{\prime} \boldsymbol{u}=\boldsymbol{x}-\left(\frac{\boldsymbol{u}^{\mathrm{H}} \boldsymbol{x}-\sqrt{b} \exp (\iota \theta)}{\|\boldsymbol{u}\|_{2}^{2}}\right) \boldsymbol{u}$. Following [59], the optimal $\theta$ that minimizes the objective $\|\boldsymbol{x}-\boldsymbol{z}\|_{2}^{2}=$ $\left\|\left(\frac{\boldsymbol{u}^{\mathrm{H}} \boldsymbol{x}-\sqrt{b} \exp (\iota \theta)}{\|\boldsymbol{u}\|_{2}^{2}}\right) \boldsymbol{u}\right\|_{2}^{2}$ is an angle of $\boldsymbol{x}^{\mathrm{H}} \boldsymbol{u}$. Thus, $\exp (\iota \theta)=$ $\frac{\boldsymbol{u}^{\mathrm{H}} \boldsymbol{x}}{\left|\boldsymbol{u}^{\mathrm{H}} \boldsymbol{x}\right|}$. Hence, the projection result follows and given in (13).

\section{APPENDIX C}

\section{DERIVATION OF SSP ALGORITHM}

We present the derivations of the SSP algorithm, whose pseudo-code is outlined in Algorithm 2 In the SSP algorithm, the set of Lagrange multipliers $\left\{\mu_{m}^{(0)}\right\}_{m=1}^{M}$ are initialized assuming that the considered $m$-th multiplier is present while others are absent, i.e., boiling down to rank 1 case where the multiplier is computed in closed-form by following [59].

Lemma 1. Let $M=1$, then a closed-form solution to the Lagrange multiplier $\mu_{m}$ is

$$
\mu_{m}=\frac{1}{\lambda_{1}^{m}}\left(\left|\boldsymbol{a}\left(\nu_{m}\right)^{\mathrm{T}} \boldsymbol{d}_{j}\right| \sqrt{\left(\frac{\lambda_{1}^{m}}{\gamma_{m}}\right)}-1\right),
$$

where $\mu \geq 0$ and $\lambda_{1}^{m}=\left\|\boldsymbol{a}\left(\nu_{m}\right)\right\|_{2}^{2}$.

Proof. Following [59, Section IIIB] and performing algebraic manipulations, we derive the closed-form solution.

After initialization, at every given iteration, we apply coordinate descent iterative scheme for the SSP algorithm that essentially utilizes the closed-form solution for rank 1 scenario.

Using Lagrangian $L\left(\overline{\boldsymbol{d}}_{j},\left\{\mu_{m}\right\}\right)$ (14), the stationarity condition of the KKT conditions, i.e., setting the gradient of $L(\cdot)$ with respect to $\overline{\boldsymbol{d}}_{j}$ to 0 , yields

$$
\overline{\boldsymbol{d}}_{j}=\left(\boldsymbol{I}_{N}+\sum_{m=1}^{M} \mu_{m} \overline{\boldsymbol{A}}_{m}\right)^{-1} \boldsymbol{d}_{j}:=\boldsymbol{G}^{-1} \boldsymbol{d}_{j} .
$$

For brevity, we define

$$
\boldsymbol{G} \equiv \boldsymbol{G}\left(\mu_{m}\right):=\left(\boldsymbol{I}_{N}+\sum_{m=1}^{M} \mu_{m} \overline{\boldsymbol{A}}_{m}\right)=\left(\boldsymbol{G}_{\backslash m}+\mu_{m} \overline{\boldsymbol{A}}_{m}\right),
$$

where $\boldsymbol{G}_{\backslash m}:=\boldsymbol{I}_{N}+\sum_{n=1 \backslash m}^{M} \mu_{n} \overline{\boldsymbol{A}}_{n}$. 
The matrix inversion of $\boldsymbol{G}\left(\mu_{m}\right)$ utilizing Sherman-Morrison formula [60] is

$$
\boldsymbol{G}\left(\mu_{m}\right)^{-1}=\left(\boldsymbol{G}_{\backslash m}^{-1}-\frac{\mu_{m} \boldsymbol{G}_{\backslash m}^{-1} \overline{\boldsymbol{A}}_{m} \boldsymbol{G}_{\backslash m}^{-1}}{1+\mu_{m} \boldsymbol{a}\left(\nu_{m}\right)^{\mathrm{T}} \boldsymbol{G}_{\backslash m}^{-1} \boldsymbol{a}\left(\nu_{m}\right)^{*}}\right),
$$

and noting the fact that the matrix $\boldsymbol{G}\left(\mu_{m}\right)$ is a sum of rank one matrices then the matrix inversion can be performed iteratively, cf. Lemma 2. In order to obtain the set of $\left\{\mu_{m}\right\}$ Lagrange multipliers, we employ coordinate descent scheme [61]. Let us say we compute $\mu_{m}$ in a given cycle, then we fix other multipliers $\mu_{n} \forall n$ but excluding $\mu_{m}$. We propose to compute and update all the $M$ Lagrange multipliers cyclically. In a given iteration cycle, we compute $\mu_{m}$ Lagrange multiplier and fixing other multipliers by utilizing the complementary slackness condition of the KKT conditions and the dual feasibility condition: 1) If $\mu_{m}=0$, then $\overline{\boldsymbol{d}}_{j}=\boldsymbol{G}_{\backslash m} \boldsymbol{d}_{j}$ (cf. (37)), and 2) If $\mu_{m}>0$, then the inequality constraint should be an equality constraint. By plugging (36) and 377) in the constraint such that

$$
\begin{aligned}
& \overline{\boldsymbol{d}}_{j}^{\mathrm{H}} \overline{\boldsymbol{A}}_{m} \overline{\boldsymbol{d}}_{j}-\gamma_{m}=\overline{\boldsymbol{d}}_{j}^{\mathrm{H}} \boldsymbol{a}\left(\nu_{m}\right)^{*} \boldsymbol{a}\left(\nu_{m}\right)^{\mathrm{T}} \overline{\boldsymbol{d}}_{j}-\gamma_{m}=0 \\
\Longleftrightarrow & \boldsymbol{a}\left(\nu_{m}\right)^{\mathrm{T}} \boldsymbol{G}\left(\mu_{m}\right)^{-1} \boldsymbol{d}_{j}=\sqrt{\gamma_{m}} \exp (\iota \phi),
\end{aligned}
$$

where $\phi$ is a free parameter. We now obtain the Lagrange multiplier $\mu_{m}$ from (39) utilizing (38) after some algebraic manipulation

$$
\begin{aligned}
& \boldsymbol{a}\left(\nu_{m}\right)^{\mathrm{T}}\left(\boldsymbol{G}_{\backslash m}+\mu_{m} \overline{\boldsymbol{A}}_{m}\right)^{-1} \boldsymbol{d}_{j}=\sqrt{\gamma_{m}} \exp (\iota \phi) \quad \text { (40) } \\
& \Longleftrightarrow\left(\boldsymbol{a}\left(\nu_{m}\right)^{\mathrm{T}} \boldsymbol{G}_{\backslash m}^{-1} \boldsymbol{d}_{j}-\mu_{m} \frac{\boldsymbol{a}\left(\nu_{m}\right)^{\mathrm{T}} \boldsymbol{G}_{\backslash m}^{-1} \overline{\boldsymbol{A}}_{m} \boldsymbol{G}_{\backslash m}^{-1} \boldsymbol{d}_{j}}{1+\mu_{m} \boldsymbol{a}\left(\nu_{m}\right)^{\mathrm{T}} \boldsymbol{G}_{\backslash m}^{-1} \boldsymbol{a}\left(\nu_{m}\right)^{*}}\right) \\
&=\sqrt{\gamma_{m}} \exp (\iota \phi) .
\end{aligned}
$$

For brevity, let $\alpha_{1}:=\boldsymbol{a}\left(\nu_{m}\right)^{\mathrm{T}} \boldsymbol{G}_{\backslash m}^{-1} \boldsymbol{d}_{j}$ and $\alpha_{2}:=$ $\boldsymbol{a}\left(\nu_{m}\right)^{\mathrm{T}} \boldsymbol{G}_{\backslash m}^{-1} \boldsymbol{a}\left(\nu_{m}\right)^{*}$ such that (41) can be rewritten as

$$
\begin{aligned}
& \alpha_{1}-\frac{\mu_{m} \alpha_{1} \alpha_{2}}{1+\mu_{m} \alpha_{2}}=\frac{\alpha_{1}}{1+\mu_{m} \alpha_{2}}=\sqrt{\gamma_{m}} \exp (\iota \phi) \\
\Rightarrow & \mu_{m}=\Re\left\{\frac{\alpha_{1} \exp (-\iota \phi)-\sqrt{\gamma_{m}}}{\sqrt{\gamma_{m}} \alpha_{2}}\right\} .
\end{aligned}
$$

\section{APPENDIX D \\ USEFUL LEMMA}

Lemma 2 (Matrix inversion with sum of rank one matrices). The matrix inversion of $\boldsymbol{A}_{r+1}=\boldsymbol{G}+\sum_{i=1}^{r} \boldsymbol{H}_{i}$ with sum of $r$ rank one matrices $\boldsymbol{H}_{i}$ can be obtained iteratively $\boldsymbol{A}_{k+1}^{-1}=$ $\boldsymbol{A}_{k}^{-1}-g_{k} \boldsymbol{A}_{k}^{-1} \boldsymbol{H}_{k} \boldsymbol{A}_{k}^{-1} \forall k=1, \ldots, r$, where $\boldsymbol{A}_{1}=\boldsymbol{G}$ and $g_{k}=1 /\left(1+\operatorname{Tr}\left(\boldsymbol{A}_{k}^{-1} \boldsymbol{H}_{k}\right)\right)$.

Proof. Let $\boldsymbol{G}$ and $\boldsymbol{G}+\boldsymbol{H}$ be invertible matrices, and $\boldsymbol{H}=$ $\sum_{i=1}^{r} \boldsymbol{H}_{i}$ with $\operatorname{rank}\left(\boldsymbol{H}_{i}\right)=1$. Let $\boldsymbol{A}_{k+1}=\boldsymbol{G}+\sum_{i=1}^{k} \boldsymbol{H}_{i}$ be invertible. By initializing $\boldsymbol{A}_{1}=\boldsymbol{G}$, then utilizing [62] result, we achieve $\boldsymbol{A}_{k+1}^{-1}=\boldsymbol{A}_{k}^{-1}-g_{k} \boldsymbol{A}_{k}^{-1} \boldsymbol{H}_{k} \boldsymbol{A}_{k}^{-1}$, for $k=1, \ldots, r$ where $g_{k}=1 /\left(1+\operatorname{Tr}\left(\boldsymbol{A}_{k}^{-1} \boldsymbol{H}_{k}\right)\right)$.

\section{ACKNOWLEDGEMENT}

This paper is dedicated to the memory of Prof. Peter Händel who prematurely passed away.

\section{REFERENCES}

[1] S. Kant, G. Fodor, M. Bengtsson, B. Göransson, and C. Fischione, "Lowcomplexity OFDM spectral precoding," in Proc. IEEE Int. Workshop Signal Process. Adv. Wireless Commun. (SPAWC), Cannes, France, 2019.

[2] E. Dahlman, S. Parkvall, and J. Sköld, 5G NR: The next generation wireless access technology. Academic Press, 2018.

[3] P. Setoodeh and S. Haykin, Fundamentals of cognitive radio. John Wiley \& Sons, Inc., 2017.

[4] R. Prasad, OFDM for wireless communications systems. Artech House, 2004.

[5] 3GPP TS 38.104, "NR; Base station (BS) radio transmission and reception," V15.9.0, 2020.

[6] X. Huang, J. A. Zhang, and Y. J. Guo, "Out-of-band emission reduction and a unified framework for precoded OFDM," IEEE Commun. Mag., vol. 53, no. 6, pp. 151-159, June 2015.

[7] M. Faulkner, "The effect of filtering on the performance of OFDM systems," IEEE Trans. Veh. Technol., vol. 49, no. 5, pp. 1877-1884, Sep. 2000.

[8] E. Bala, J. Li, and R. Yang, "Shaping spectral leakage: A novel lowcomplexity transceiver architecture for cognitive radio," IEEE Veh. Technol. Mag., vol. 8, no. 3, pp. 38-46, July 2013.

[9] H. Yamaguchi, "Active interference cancellation technique for MBOFDM cognitive radio," in Proc. IEEE Eur. Microw. Conf., 2004, pp. $1105-1108$

[10] S. Brandes, I. Cosovic, and M. Schnell, "Sidelobe suppression in OFDM systems by insertion of cancellation carriers," in Proc. IEEE Veh. Technol. Conf., 2005, pp. 152-156.

[11] D. Qu, Z. Wang, and T. Jiang, "Extended active interference cancellation for sidelobe suppression in cognitive radio OFDM systems with cyclic prefix," IEEE Trans. Veh. Technol., vol. 59, no. 4, pp. 1689-1695, May 2010.

[12] I. Cosovic, S. Brandes, and M. Schnell, "Subcarrier weighting: a method for sidelobe suppression in OFDM systems," IEEE Commun. Lett., vol. 10, no. 6, pp. 444-446, June 2006.

[13] J. van de Beek, "Sculpting the multicarrier spectrum: A novel projection precoder," IEEE Commun. Lett., vol. 13, no. 12, pp. 881-883, 2009.

[14] H. Chen, W. Chen, and C. Chung, "Spectrally precoded OFDM and OFDMA with cyclic prefix and unconstrained guard ratios," IEEE Trans. Wireless Commun., vol. 10, no. 5, pp. 1416-1427, May 2011.

[15] A. Tom, A. Sahin, and H. Arslan, "Mask compliant precoder for OFDM spectrum shaping," IEEE Commun. Lett., vol. 17, no. 3, pp. 447-450, 2013.

[16] R. Kumar and A. Tyagi, "Weighted least squares based spectral precoder for OFDM cognitive radio," IEEE Wireless Commun. Lett., vol. 4, no. 6, pp. 641-644, Dec. 2015.

[17] M. Mohamad, R. Nilsson, and J. van de Beek, "A novel transmitter architecture for spectrally-precoded OFDM," IEEE Trans. Circuits Syst. I: Regular Papers, vol. 65, no. 8, pp. 2592-2605, Aug. 2018.

[18] M. Mohamad, "Spectrally precoded OFDM design and analysis," $\mathrm{PhD}$ dissertation, Department of Computer Science, Electrical and Space Engineering, Luleå University, Luleå, Sweden, 2019.

[19] P. L. Combettes and J.-C. Pesquet, "Proximal splitting methods in signal processing," in Fixed-Point algorithms for inverse problems in science and engineering. Springer, New York, NY, 2011, pp. 185-212.

[20] S. Boyd, N. Parikh, E. Chu, B. Peleato, and J. Eckstein, "Distributed optimization and statistical learning via the alternating direction method of multipliers," Foundations and Trends ${ }^{\circledR}$ in Machine Learning, vol. 3, no. 1, pp. 1-122, 2011.

[21] J. Eckstein and D. P. Bertsekas, "On the Douglas-Rachford splitting method and the proximal point algorithm for maximal monotone operators," Mathematical Programming, vol. 55, no. 1-3, pp. 293-318, Apr. 1992.

[22] D. M. Pozar, Microwave Engineering, 4th Edition. Wiley, 2011.

[23] M. Mohamad, R. Nilsson, and J. van de Beek, "Minimum-EVM Ncontinuous OFDM," in Proc. 2016 IEEE Int. Conf. on Commun. (ICC), May 2016, pp. 1-5.

[24] R. Kumar and A. Tyagi, "Computationally efficient mask-compliant spectral precoder for OFDM cognitive radio," IEEE Trans. on Cogn. Commun. Netw., vol. 2, no. 1, pp. 15-23, 2016.

[25] S. P. Boyd and L. Vandenberghe, Convex optimization. Cambridge University Press, 2004.

[26] J. van de Beek and F. Berggren, "EVM-constrained OFDM precoding for reduction of out-of-band emission," in Proc. IEEE Veh. Tech. Conf. Fall, Sep. 2009, pp. 1-5. 
[27] R. Pitaval and B. M. Popovi, "Linear receivers for spectrally-precoded MIMO-OFDM," IEEE Commun. Lett., vol. 21, no. 6, pp. 1269-1272, June 2017.

[28] N. N. Moghadam, P. Zetterberg, P. Händel, and H. Hjalmarsson, "Correlation of distortion noise between the branches of MIMO transmit antennas," in Proc. IEEE Int. Symp. Pers., Indoor, Mobile Radio Commun. (PIMRC), Sep. 2012, pp. 2079-2084.

[29] E. Sienkiewicz, N. McGowan, B. Göransson, T. Chapman, and T. Elfström, "Spatially dependent ACLR modelling," in Proc. IEEE Conf. Antenna Meas. Appl. (CAMA), Nov. 2014, pp. 1-4.

[30] E. G. Larsson and L. Van Der Perre, "Out-of-band radiation from antenna arrays clarified," IEEE Wireless Commun. Lett., vol. 7, no. 4, pp. 610-613, Aug. 2018.

[31] N. N. Moghadam, G. Fodor, M. Bengtsson, and D. J. Love, "On the energy efficiency of MIMO hybrid beamforming for millimeterwave systems with nonlinear power amplifiers," IEEE Trans. Wireless Commun., vol. 17, no. 11, pp. 7208-7221, Nov. 2018.

[32] G. Fettweis, M. Lohning, D. Petrovic, M. Windisch, P. Zillmann, and W. Rave, "Dirty RF: a new paradigm," in Proc. IEEE Int. Symp. Pers., Indoor, Mobile Radio Commun. (PIMRC), vol. 4, 2005, pp. 2347-2355 Vol. 4.

[33] T. Schenk, RF imperfections in high-rate wireless systems. The Netherlands: Springer, 2008.

[34] B. Göransson, S. Grant, E. Larsson, and Z. Feng, "Effect of transmitter and receiver impairments on the performance of MIMO in HSDPA," in Proc. IEEE Works. on Sign. Proc. Adv. in Wireless Commun., 2008, pp. 496-500.

[35] C. Studer, M. Wenk, and A. Burg, "MIMO transmission with residual transmit-RF impairments," in Proc. ITG Workshop Smart Antennas, Feb. 2010, pp. 189-196.

[36] _ "System-level implications of residual transmit-RF impairments in MIMO systems," in Proc. European Conf. on Antennas and Propagation, Apr. 2011, pp. 2686-2689.

[37] E. Björnson, J. Hoydis, and L. Sanguinetti, "Massive MIMO networks: Spectral, energy, and hardware efficiency," Foundations and Trends®in Signal Processing, vol. 11, no. 3-4, pp. 154-655, 2017.

[38] E. Björnson, J. Hoydis, M. Kountouris, and M. Debbah, "Massive MIMO systems with non-ideal hardware: Energy efficiency, estimation, and capacity limits," IEEE Trans. Inf. Theory, vol. 60, no. 11, pp. 7112 7139, Sep. 2014.

[39] A. A. A. Boulogeorgos and A. Alexiou, "Analytical performance evaluation of beamforming under transceivers hardware imperfections," in Proc. IEEE Wireless Commun. and Networking Conf., 2019, pp. 1-7.

[40] R. Hassun, M. Flaherty, R. Matreci, and M. Taylor, "Effective evaluation of link quality using error vector magnitude techniques," in Proc. IEEE Wireless Commun. Conf., 1997, pp. 89-94.

[41] H. A. Mahmoud and H. Arslan, "Error vector magnitude to SNR conversion for nondata-aided receivers," IEEE Trans. Wireless Commun., vol. 8, no. 5, pp. 2694-2704, May 2009.

[42] T. L. Jensen and T. Larsen, "Robust computation of error vector magnitude for wireless standards," IEEE Trans. Commun., vol. 61, no. 2 , pp. 648-657, Mar. 2013.

[43] 3GPP TS 38.101-4, "User equipment (UE) radio transmission and reception; part 4: Performance requirements," V15.5.0, 2020.

[44] 3GPP TS 38.214, "NR; Physical layer procedures for data," V15.9.0, 2020.

[45] R. A. Shafik, M. S. Rahman, and A. R. Islam, "On the extended relationships among EVM, BER and SNR as performance metrics," in Proc. Int. Conf. Elect. Comput. Eng., 2006, pp. 408-411.

[46] K. Freiberger, "Measurement methods for estimating the error vector magnitude in OFDM transceivers," PhD dissertation, Doctoral School of Information and Communication Engineering, Graz University of Technology, Austria, 2017.

[47] M. Grant and S. Boyd, "CVX: Matlab software for disciplined convex programming, version 2.1," http://cvxr.com/cvx Mar. 2014.

[48] N. Parikh, S. Boyd, N. Parikh, and S. Boyd, "Proximal algorithms," Foundations and Trends® in Optimization, vol. 1, no. 3, pp. 123-231, 2013.

[49] A. Beck, First-order methods in optimization. SIAM, 2017.

[50] Z. Q. Luo and P. Tseng, "On the convergence of the coordinate descent method for convex differentiable minimization," Journal of Optimization Theory and Applications, vol. 72, no. 1, pp. 7-35, Jan. 1992.

[51] C.-Y. Chi, W.-C. Li, and C.-H. Lin, Convex optimization for signal processing and communications: from fundamentals to applications, 1st ed. CRC Press, 2017.
[52] P. L. Combettes, "Solving monotone inclusions via compositions of nonexpansive averaged operators," Optimization, vol. 53, no. 5-6, pp. 475-504, Oct. 2004.

[53] Y. Liu, E. K. Ryu, and W. Yin, "A new use of Douglas-Rachford splitting for identifying infeasible, unbounded, and pathological conic programs," Computational Optimization and Applications, vol. 177, p. 225-253, Sep. 2019.

[54] E. K. Ryu, Y. Liu, and W. Yin, "Douglas-Rachford splitting and ADMM for pathological convex optimization," Computational Optimization and Applications, vol. 74, p. 747-778, Sep. 2019.

[55] 3GPP TS 38.212, "NR; Multiplexing and channel coding," V15.8.0, 2020.

[56] 3GPP TS 34.121, "Terminal conformance specification, radio transmission and reception (FDD)," V6.4.0, 2006.

[57] 3GPP TS 38.306, "NR; User equipment (UE) radio access capabilities," V15.9.0, 2019.

[58] 3GPP TS 38.211, "NR; Physical channels and modulation," V15.8.0, 2020.

[59] K. Huang and N. D. Sidiropoulos, "Consensus-ADMM for general quadratically constrained quadratic programming," IEEE Trans. Signal Process., vol. 64, no. 20, pp. 5297-5310, Oct. 2016.

[60] C. D. Meyer, Matrix analysis and applied linear algebra. Society for Industrial and Applied Mathematics, 2000.

[61] P. Tseng, "Convergence of a block coordinate descent method for nondifferentiable minimization," Journal of Optimization Theory \& Applications, vol. 109, no. 3, pp. 475-494, June 2001.

[62] K. S. Miller, "On the inverse of the sum of matrices," Mathematics Magazine, vol. 54, no. 2, pp. 67-72, Mar. 1981. 